\title{
芳香偶氮衍生物合成策略研究的新进展
}

\author{
钦传光*,a,b 李 洋 $b$ 李海亮 $b$ 李大为 $b$ \\ 牛卫宁 ${ }^{b}$ 尚晓娅 ${ }^{b} \quad$ 徐春兰 ${ }^{b}$ \\ $\left({ }^{a}\right.$ 西北工业大学理学院应用化学系 教育部空间应用物理与化学重点实验室 \\ 陕西省高分子科学与技术重点实验室 西安 710129) \\ ( ${ }^{b}$ 西北工业大学生命学院 西安 710072)
}

\begin{abstract}
摘要 芳香偶氮化合物具有独特的光致顺反异构特性，不仅应广泛用于传统化学工业，还将应用于光化学分子开关、 主客体超分子化学识别、自组装液晶材料、生物医学成像与化学分析以及光驱分子马达等诸多新兴科学领域. 特别具 挑战性的是开发具有高化学稳定性和热稳定性又易检测的偶氮发色团，近年来受到科研工作者们的高度关注. 随着人 们对研究新型芳香偶氮衍生物的迫切需要，又相继创新和发展了一些更新、更有效的芳香偶氮化合物合成方法，综述 了最近新型芳香偶氮衍生物合成方法的新进展，尤其强调了芳基肼的氧化脱氢反应和金属催化偶联反应、芳胺的氧化 反应、硝基芳香化合物的还原偶联反应、芳香偶氮氧化物的转化与还原、叠氮芳香化合物的催化偶联与热分解反应以 及芳香基重氮盐的偶合与催化偶联反应等在芳香偶氮化合物合成方面应用的新趋势.
\end{abstract}

关键词 芳香偶氮衍生物; 合成策略; 芳基肼; 芳胺; 芳香硝基化合物; 芳香叠氮化合物

\section{Novel Progresses in Synthesis Strategies of Aromatic Azo Derivatives}

\author{
Qin, Chuanguang*,a,b \\ Li, Yang ${ }^{b}$ \\ Li, Hailiang ${ }^{b}$ \\ Li, Dawei ${ }^{b}$ \\ Niu, Weining ${ }^{b} \quad$ Shang, Xiaoya ${ }^{b} \quad$ Xu, Chunlan ${ }^{b}$
}

( ${ }^{a}$ Key Laboratory of Space Applied Physics and Chemistry of Ministry of Education and Key Laboratory of Macromolecular Science \& Technology of Shaanxi Province, Department of Applied Chemistry, School of Natural \& Applied Sciences, Northwestern Polytechnical University, Xi'an 710129)

$\left({ }^{b}\right.$ School of Life Science, Northwestern Polytechnical University, Xi'an 710072)

\begin{abstract}
Aromatic azo derivatives possess a particular character that is the light driven reversible isomerization between their cis- and trans-forms, which makes them excellent candidates to modulate the relative movement of different moieties. They are widely used not only in the traditional chemical industry, but also in many newly rising areas of science, such as photochemical molecular switch, super-molecular chemistry of host-guest recognition, self-assembly liquid crystal material, analysis of biomedical imaging and chemical, light driven molecular motor, and so on. The development of chromophores with characteristics, like high chemical stability, thermal stability over a wide temperature range and having two forms easily detectable by a method that does not cause irreversible molecular alterations, is a major challenge for researchers who focus their interest in this area. With the increasing need for investigating unknown aromatic azo derivatives, many novel efficient synthesis strategies are recently innovated and developed in succession. This critical review covers the various synthetic methods of aromatic azo derivatives reported more recently, with special emphasis on application of arylhydrazine, arylamine, nitroarene, azoxyarene, arylazide, and their derivatives.
\end{abstract}

Keywords azoarene derivative; synthesis strategy; arylhydrazine; arylamine; nitroarene; arylazide

\footnotetext{
*E-mail: qinchg@nwpu.edu.cn

Received September 28, 2012; revised November 15, 2012; published online November 20, 2012
}

Project supported by the National Natural Science Foundation of China (Nos. 31001012, 31101304, 20802057, 20672086), the Natural Science Basic Research Plan in Shaanxi Province of China (No. 2012JZ2002) and the Postgraduate Pioneer Seed Funding of Northwestern Polytechnical University (No. Z2012198). 国家自然科学基金(Nos. 31001012, 31101304, 20802057, 20672086)、陕西省自然科学基础研究计划重点(No. 2012JZ2002)和西北工业大学研究生创业 种子基金(No. Z2012198)资助项目. 
芳香偶氮化合物在传统的化学工业中不仅作为染 料、色素 ${ }^{[1]}$ 、食品添加剂、指示剂 ${ }^{[2]}$ 、自由基反应诱导 剂 $^{[3]}$ 及治疗药物 ${ }^{[4]}$ 等有着广泛的应用. 而且在药物传 递 ${ }^{[5]}$ 和光电子学 ${ }^{[6]}$ 方面也显示出较好的前景. 特别是由 于芳香偶氮化合物具有在光和热的作用下可以发生顺 反异构现象的特性和优良的光致变色性能, 其在现代高 技术领域中的研究也开始倍受人们的青睐, 相继在非线 性光电信息材料 ${ }^{[7]}$ 、光学存储介质 ${ }^{[8]}$ 、化学传感器 ${ }^{[9]}$ 、液 晶材料 ${ }^{[10,11]}$ 、光化学分子开关 ${ }^{[12,13]}$ 、生物荧光显影成像 剂 ${ }^{[14]}$ 、分子机器 ${ }^{[15]}$ 、纳米管 ${ }^{[16]}$ 和防护眼镜过滤器 ${ }^{[17]}$ 的 制造等领域有广泛的应用潜力. 此外, 光驱动偶氮苯顺 式和反式之间可逆的异构化使它们成为调节不同基团 相对运动的极佳的候选者. 一个含有偶氮苯基团的分子 的运动能够控制非共价结合在偶氮苯片段上的互补底 物的运动 ${ }^{[18]}$. 在生物系统中, 偶氮化合物的感光反应改 变酶和多肽类的构象和活性 ${ }^{[19]}$. 芳香偶氮化合物也引 起人们对阿尔兹海默尔疾病的更精确诊断的兴趣, 因为 它们在生理上可以被用作探针使精神恶化病人脑内的 淀粉样蛋白小半鞘翅可视化 ${ }^{[20]}$. 因此, 关于芳香偶氮衍 生物的合成方法及其功能化应用研究, 近年来再度成为 学术界引人关注的热点. 众所周知, 芳香偶氮化合物的 经典合成反应是重氮化偶合反应, 米尔斯和瓦拉赫反 应. 随着人们对研制新型芳香偶氮衍生物的迫切需要, 最近又相继发展了一些更新、更有效的芳香偶氮化合物 合成方法, 本文综述了最近新型芳香偶氮衍生物合成方 法的新进展，尤其强调了芳基肼的氧化脱氢反应和金属 催化偶联反应、芳胺的氧化反应、硝基芳香化合物的还 原偶联反应、芳香偶氮氧化物的转化与还原、叠氮芳香 化合物的催化偶联与热分解反应以及芳香基重氮盐的 偶合与催化偶联反应等在芳香偶氮化合物合成方面应 用的新趋势.

\section{1 芳香偶氮衍生物合成方法的创新与发展}

\section{1 芳基肼的氧化脱氢反应和金属催化偶联}

通过相应的 $N, N^{\prime}$-二芳基肼的氧化脱氢可以获得芳 香偶氮化合物, 最近采用化学计量的氧化剂, 包括聚乙 烯亚胺树脂高碘酸盐, $\mathrm{HgO}, \mathrm{MnO}_{2}, \mathrm{O}_{2}$ 或 $\mathrm{H}_{2} \mathrm{O}_{2}, \mathrm{NaBrO}_{3} /$ $\mathrm{H}_{2} \mathrm{SO}_{4}$, 空气 $\left(\mathrm{O}_{2}\right) / \mathrm{NaOH}, \mathrm{Pb}(\mathrm{OAc})_{4},\left(\mathrm{NH}_{4}\right)_{2} \mathrm{~S}_{2} \mathrm{O}_{8}$, 硝酸铈 (IV)四丁胺铵, $\mathrm{K}_{3} \mathrm{Fe}(\mathrm{CN})_{6} / 2,4,6$-三苯基苯酚或 $\mathrm{CuCl}_{2} /$ 2,4,6-三叔丁基苯酚, $(\mathrm{PhSeO})_{2} \mathrm{O}$ 或 $\mathrm{PhSeO}_{2} \mathrm{H}$, 芳基磺酰 过氧化物, $\mathrm{NaNO}_{2}$ /醋酸䣶以及硅胶承载 $\mathrm{NaNO}_{2} / \mathrm{NaHSO}_{4}$ 等等. 此外, 单芳基肼也可以被某些有机化合物如苯醌 及其缩醛氧化成芳香偶氮化合物. 一般来说, 通过氧化 脱氢作用在较短的时间内 $(5 \mathrm{~min} \sim 2 \mathrm{~h})$ 产率比较好 (72\% 99\%). 利用含有聚乙烯亚胺树脂高碘酸盐从二
苯肼 1 合成偶氮苯 2 , 产率 $88 \%$ (Eq. 1) ${ }^{[21]}$.

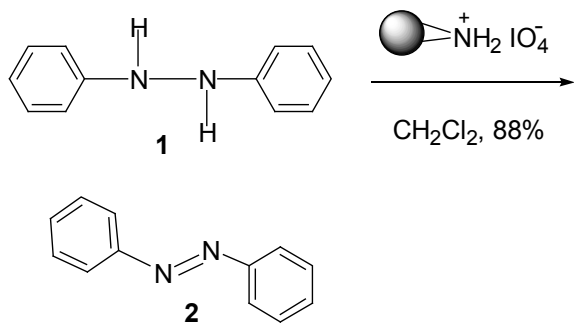

他们为了获得偶氮化合物 6 作为光开关交联剂研究 多肽或蛋白质的构象和功能受光调控的变化情况, 先将 硝基化合物 3 经还原制备得到桥环芳基肼 4 , 再采用 $\mathrm{HgO}$ 在乙醇中氧化桥环芳基肼 $\mathbf{4}$ 获得桥环偶氮化合物 $\mathbf{5}$ 作为合成目标产物 $\mathbf{6}$ 的前体原料 ${ }^{[22]}$ (Scheme 1).

在室温和有光存在的时候, 二氧化锰被用作氧化剂 引发邻位或 3-取代二苯肼(7), 以较高的产率 $(86 \%$ $98 \%$ ) 获得相应的顺式偶氮苯 $\mathbf{8}^{[23]}$. 另外, 顺式偶氮苯 $\mathbf{8}$ 热异构化成反式偶氮苯 9 发生的温度在 $70{ }^{\circ} \mathrm{C}$ 以上 (Scheme 2). 当 4-取代位上连接有强共轭基团如 NHAc, $\mathrm{OAc}, \mathrm{OMe}$ 等的 $N, N$-二苯基肼系统时, 通常只能获得其 反式偶氮苯, 而得不到相应的顺式偶氮苯.

用氧或双氧水作为氧化剂, $\mathrm{NH}_{4} \mathrm{VO}_{3}$ 和钴络合物能 有效地催化这种氧化转化. 而且, 当 $\mathrm{KClO}_{3} / \mathrm{H}_{2} \mathrm{SO}_{4}$ 混合 物被用作氧化剂时, 硫酸亚铁显示出能充当次联氨基氧 化催化剂. $\mathrm{TiCl}_{3} / \mathrm{HBr}$ 系统和 $\mathrm{H}_{2} \mathrm{O}_{2}$ 作为氧化剂能催化次 联氨基衍生物氧化成相应磺酰基偶氮化合物, 且有较高 的产率, 这个系统比 $\mathrm{NH}_{4} \mathrm{VO}_{3}$ 更有效. Gozin 等 ${ }^{[24]}$ 采用这 类反应将二芳基肼 10 转化成芳香偶氮化合物 11, 并对 比研究了不同催化剂对于不同取代化合物的转化效率 (Eq. 2).

牛永生等 ${ }^{[25]}$ 采用价廉易得的氧化剂 $\mathrm{NaBrO}_{3}$ 和 $\mathrm{H}_{2} \mathrm{SO}_{4}$, 在温和的条件下, 氧化取代芳酰肼类化合物 2a $\sim 12 \mathrm{~m}$ (Eq. 3)，方便高效地合成了相应的 13 种芳酰 基偶氮化合物 13a 13m, 产率 84\% 94\%. 用红外光 谱、核磁共振氢谱及元素分析等技术方法对所合成的产 物进行了结构表征, 并讨论了可能的反应机理.

2003 年 Cho 等 ${ }^{[26]}$ 报道了钯催化 $N$-Boc-芳基肼和芳 基卤化物偶联反应作为合成偶氮化合物的一种新的方 法, 合成的二芳基肼在二氯甲烷中和 $\mathrm{NBS}$ /吡啶在室温 下直接氧化成偶氮苯，产率较好，可以达到 $60 \%$ 左右. 在随后的研究中, 进一步发现了用 $\operatorname{Pd}(\mathrm{OAc})_{2}$ 催化偶联 Boc-芳肼 14, 19 与芳基卤化物 15, 20 以获得 Boc-二芳 基肼 16, 21, 在碘化亚铜/碱或 NBS/吡啶存在的条件下, 它们被氧化成芳香偶氮化合物 17,22 , 产率 $60 \%$ 以上 (Scheme 3) ${ }^{[27,28]}$. 这种反应的机制还没有被完全阐明. 


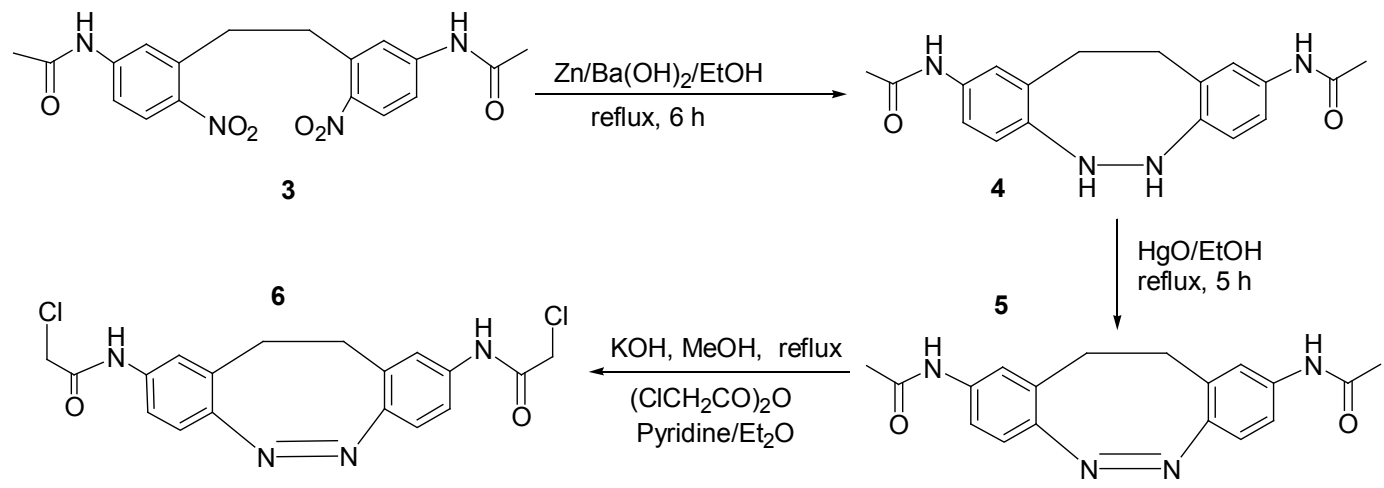

Scheme 1

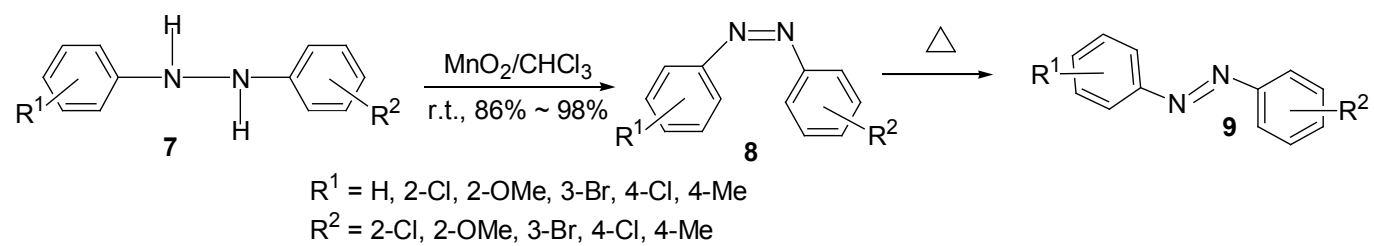

Scheme 2

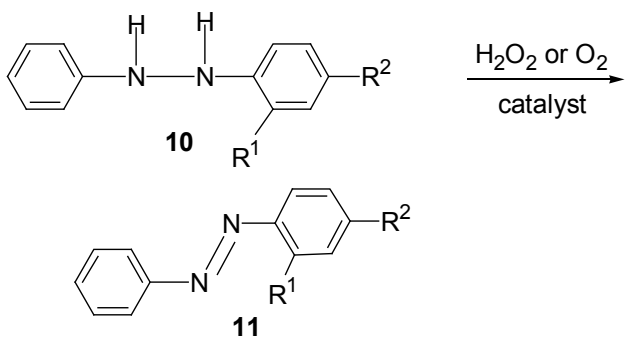

\begin{tabular}{cccc}
\hline \multirow{2}{*}{$\mathrm{R}^{1}$} & \multirow{2}{*}{$\mathrm{R}^{2}$} & \multicolumn{2}{c}{ Yield/\% } \\
\cline { 3 - 4 } & & $\mathrm{TiCl}_{3} / \mathrm{HBr}$ & $\mathrm{NH}_{4} \mathrm{VO}_{3}$ \\
\hline $\mathrm{H}$ & $\mathrm{H}$ & 95 & 96 \\
$\mathrm{NO}_{2}$ & $\mathrm{NO}_{2}$ & 88 & 47 \\
$\mathrm{H}$ & $\mathrm{OMe}$ & 95 & 82 \\
\hline
\end{tabular}
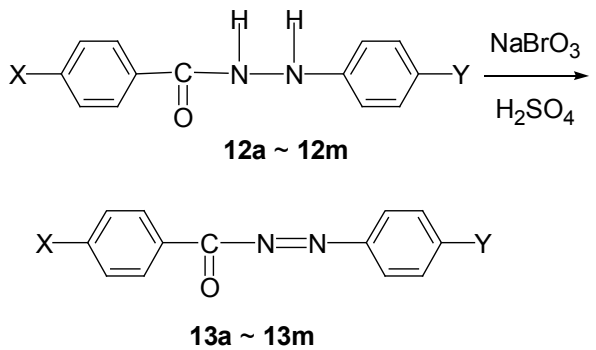

\begin{tabular}{lccccccccccccc}
\hline & $\mathbf{a}$ & $\mathbf{b}$ & $\mathbf{c}$ & $\mathbf{d}$ & $\mathbf{e}$ & $\mathbf{f}$ & $\mathbf{g}$ & $\mathbf{h}$ & $\mathbf{i}$ & $\mathbf{g}$ & $\mathbf{k}$ & $\mathbf{l}$ & $\mathbf{m}$ \\
\hline $\mathrm{X}$ & $\mathrm{OMe}$ & $\mathrm{OMeOMe}$ & $\mathrm{H}$ & $\mathrm{OEt}$ & $\mathrm{Br}$ & $\mathrm{OEt}$ & $\mathrm{Br}$ & $\mathrm{H}$ & $\mathrm{OEt}$ & $\mathrm{Br}$ & $\mathrm{OEt}$ & $\mathrm{Br}$ \\
$\mathrm{Y}$ & $\mathrm{NO}_{2}$ & $\mathrm{Cl}$ & $\mathrm{Br}$ & $\mathrm{NO}_{2}$ & $\mathrm{NO}_{2}$ & $\mathrm{NO}_{2}$ & $\mathrm{Cl}$ & $\mathrm{Cl}$ & $\mathrm{Br}$ & $\mathrm{Br}$ & $\mathrm{Br}$ & $\mathrm{H}$ & $\mathrm{H}$ \\
\hline
\end{tabular}

认为氧化步骤是通过 Boc 基团最初消除进行的. 通 过薄层色谱法, 起初形成的 $N$-Boc-二芳基肼具有较大的 极性, 它的结构可能和对应. 单 Boc 保护的二芳基肼在 转变成更小极性的偶氮化合物之前已经被从随后的水 解反应中分离出来 ${ }^{[29]}$.
已经有研究者 ${ }^{[30]}$ 报道了在酸性介质中, 苯肼 23 与 苯醌 $\mathbf{2 4}$ 反应生成偶氮化合物 $\mathbf{2 5}$, 产率 $\mathbf{7 7 \%}$ (Scheme 4). 1963 年, Hecker 和 Lattrell ${ }^{[31]}$ 报道了采用醋酸铊(III)氧化 烷基苯酚生成4-烷基-4-差基二羰基环己烷的方法，这些 化合物在乙酸或者硫酸存在的情况下与 2,4-二硝基苯肼 反应, 以较低产率生成相应的偶氮苯. McKillop 和 Taylor 等 ${ }^{[2]}$ 用类似的方法在甲醇中用硝酸铊(III)氧化 4对甲氧酚 26 得到对苯醌的单缩酮 27 , 然后在酸 $\left(\mathrm{BF}_{3}-\mathrm{Et}_{2} \mathrm{O}\right)$ 存在的情况下加入芳肼 $\mathbf{2 8}$ 形成腙, 随即在自 发消除 $\mathrm{MeOH}$ 进行芳构化的过程中丢失 $\mathrm{C}=\mathrm{N}$ 键 $\alpha$ 位上 的一个酸质子, 获得偶氮化合物 29 , 产率 $99 \%$. 另外一 个例子是在硫酸的催化作用下, 2,4 -二硝基苯肼 $(\mathbf{2 8})$ 与对 苯醌冠醚 30 反应生成偶氮苯基冠醚 $\mathbf{3 1}$, 产率 $81 \%$ (Eq. 4) ${ }^{[33]}$.

2004 年, Carreno 等 ${ }^{[34]}$ 报告了通过 1,4-二甲氧基阳极 氧化可轻易获得的苯醌缩醛类 33a 33c 与取代芳基肼 32a $\sim 32 \mathrm{k}$ 反应生成偶氮苯 34, 有较好的产率(Eq. 5). 催 化计量的铈硝酸铵 $\left\{\left(\mathrm{NH}_{4}\right)_{2}\left[\mathrm{Ce}\left(\mathrm{NO}_{3}\right)_{6}\right], \mathrm{CAN}\right\}$ 的存在能缩 短反应时间. 当二乙缩醛核心有一个取代基时，仅仅获 得一个结构异构体. 他们随后报道了选用苯肼 32c, 32f, 32g, 32i 与对位甲苯基-亚磺酰基功能化的对位二醌缩 酮 33c 反应，位置区域选择性地合成了光化学纯的偶氮 苯 34 .

通过圆二色性对他们的光敏感行为的研究表明偶 氮苯上硫氧化物的位置造成了两个不同手性反应 ${ }^{[35,36]}$. 因此，在顺反异构体的 C-3 上存在的对甲苯基亚磺酰基 基团诱导手性从立体的硫上向 $\mathrm{N}=\mathrm{N}$ 基转移. 然而, 当 C-2 位上有硫氧化物时, 手性的转移只发生在顺式异构 
<smiles>CC(C)(C)OC(=O)N(N)c1cccc(-c2cccc(-c3cccc(I)c3)c2)c1</smiles>

$\mathrm{Pd}(\mathrm{OAc})_{2}$ $\stackrel{t-\mathrm{Bu}_{3} \mathrm{P}}{\longrightarrow}$ Toluene $54 \%$<smiles>CC(C)(C)OC(=O)N1CNc2cccc(c2)-c2cccc(c2)N(C(=O)c2ccccc2)c2cccc(c2)-c2cccc(c2)N1C(=O)c1ccccc1</smiles><smiles>CC(C)(C)C(=O)O</smiles>
$\overrightarrow{D M F}, 140^{\circ} \mathrm{C}$ $60 \%$<smiles>c1cc2cc(c1)/N=N/c1cccc(c1)-c1cccc(c1)-c1cccc(c1)/N=N/2</smiles>

17

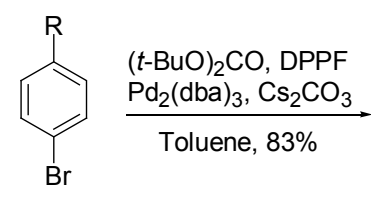

18<smiles>[R]c1ccc(N(N)C(=O)OC(C)(C)C)cc1</smiles>

19
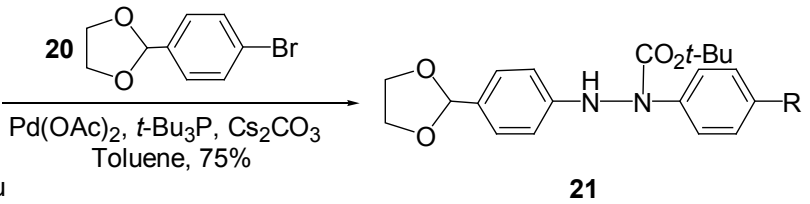

21
$\underset{\mathrm{NBS}, \text { Pyridine }}{\mathrm{CH}_{2} \mathrm{Cl}_{2}, 61 \%}$

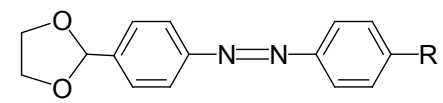

22

Scheme 3<smiles>NNc1ccc([N+](=O)[O-])cc1</smiles>

23<smiles>CC1=CC(=O)C=C(C)C1=O</smiles>

24<smiles>Cc1cc(N=C=Nc2ccc([N+](=O)[O-])cc2)cc(C)c1O</smiles>

25<smiles>CCCOC1(OC)C=CC(=O)C=C1COc1ccc([N+](=O)[O-])c(NN)c1</smiles>

Scheme 4<smiles></smiles>

30

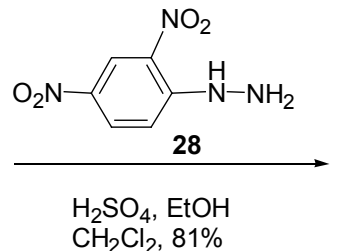

$\mathrm{CH}_{2} \mathrm{Cl}_{2}, 81 \%$
体中. 正如 NMR 研究表明, 这种不同的光学反应是由 构象被硫氧化物固定的结果. 在偶氮苯上选择合适的硫 氧化物的位置, 受控制的光照能诱导构象的变化. 当反 式异构体向顺式异构体 $\mathrm{N}=\mathrm{N}$ 双键转变时, 可以控制系 统地全部处理. 用三乙胺抑制反应表明, 酸性物质的存 在可能触发反应的进行. 可能的激活剂是被空气氧化的 芳肼的单电子氧化物提供的自由基正离子. 这种酸性物<smiles>O=[N+]([O-])c1ccc(N=NC2C=C(COC(c3ccccc3)c3ccccc3)C(O)C(COC(COCCOCCOCC(c3ccccc3)c3ccccc3)[N+](=O)[O-])=C2)c([N+](=O)[O-])c1</smiles> 

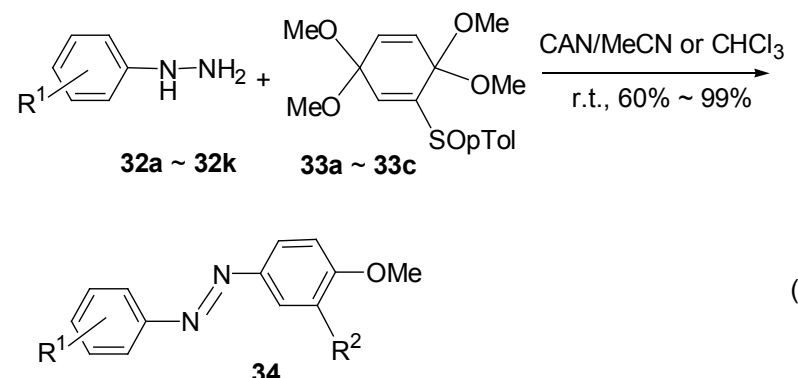

$\mathrm{R}^{1}=\mathrm{H}, p-\mathrm{OMe}, 2,4-\left(\mathrm{NO}_{2}\right)_{2}, o-\mathrm{NO}_{2}, p-\mathrm{NO}_{2}, o-\mathrm{Br}, p-\mathrm{Br}, o-\mathrm{CF}_{3}$, $2,5-\mathrm{F}_{2}, 2,3,4,5,6-\mathrm{F}_{5}, 2,3,5,6-\mathrm{F}_{4}-4-\mathrm{CF}_{3}$

$\mathrm{R}^{2}=\mathrm{H}, \mathrm{OMe}, \mathrm{SO} p-\mathrm{Tol}$

\section{2 芳胺的氧化反应}

早在 1972 年, Wawzonek 和 McIntyre 就报道了芳香 胺的电解氧化作为一种新的获得偶氮苯的方法. 然而, 这种方法产生的偶氮化合物的产率较低. 因此, 例如在 二甲基甲酰胺中, 在吡啶存在的情况下, 通过在 Pt 电极 上电解氧化 2,4,6-三氯苯胺获得相应的偶氮苯, 最高产 率只有 $48 \%$. 其后, 又报道了一系列金属的或非金属的 试剂, 例如 $\mathrm{Ag}_{2} \mathrm{CO}_{3}, \mathrm{Ag}_{2} \mathrm{O}, \mathrm{AgO}, \mathrm{AgMnO}_{4}, \mathrm{MnO}_{2}, \mathrm{KO}_{2}$, $\mathrm{NaBO}_{3}, \mathrm{~Pb}(\mathrm{OAc})_{4}, \mathrm{BaMnO}_{4}, \mathrm{Ce}(\mathrm{OH})_{3} \mathrm{O}_{2} \mathrm{H}$, 二-(2,2'-双吡 啶基)铜(II), 高锰酸, 过氧化镍, 双嘧啶, 高锰酸银, $\mathrm{RuCl}_{3} / \mathrm{H}_{2} \mathrm{O}_{2}$, 多价碘试剂, 如[PhI $\left.(\mathrm{OAc})_{2}\right], \mathrm{Mn}$ (四苯基卟 啉) $\mathrm{Cl} / \mathrm{NaIO}_{4}$, galvinoxyl 自由基 $/ \mathrm{K}_{3} \mathrm{Fe}(\mathrm{CN})_{6}$, 或者在不同 条件下的需氧氧化 $\left(\mathrm{O}_{2} / \mathrm{KO} t-\mathrm{Bu}, \mathrm{O}_{2} / \mathrm{CO}_{3} \mathrm{O}_{4}\right.$, 过氧化物酶/ $\mathrm{H}_{2} \mathrm{O}_{2}$ 和 $\mathrm{O}_{2} / \mathrm{CuCl}$, 被化学计量地或者过量地用于从芳香 胺合成偶氮苯, 通常获得偶氮化合物的产率较低或者适 中 ${ }^{[38]}$. 人们后来还发现了其他不同的产生偶氮化合物 的氧化剂像 $\left[\mathrm{C}_{16} \mathrm{H}_{33} \mathrm{~N}\left(\mathrm{CH}_{3}\right)_{3}\right]_{2} \mathrm{Cr}_{2} \mathrm{O}_{7}$ 或者五水硫酸铜承载 高锰酸钾 ${ }^{[39]}$, 过硼酸钠/醋酸 ${ }^{[40]}$. 最近, Orito 等 ${ }^{[41]}$ 报道 $\mathrm{HgO}-\mathrm{I}_{2}$ 氧化乙基-4-氨基苯甲酸酯使偶氮化合物的产率 达 $87 \%$, 反应涉及初步形成的阳离子自由基 I 或 II 得 $\mathrm{N}-\mathrm{N}$ 连接, 可能是通过一个电子转移而产生, 然后, 合 成的氢化偶氮苯的两个电子氧化获得相应的偶氮苯. 这 种试剂也被用于在室温条件下, 苯胺 $35 \mathrm{a} \sim 35 \mathrm{z}$ 光化学 氧化获得较高产率的对称的偶氮苯 $36 \mathbf{a} \sim 36 z(E q .6)^{[42]}$. 对称偶氮化合物的光化学合成的机制涉及因一个电子 转移产生的苯胺自由基的 $\mathrm{N}-\mathrm{N}$ 键连接, 然后氢化偶氮 苯的两个电子氧化形成偶氮化合物. 作者提出, 光激氧 化永使氢原子从氨基中分离, 然后快速的电子转移形成 元素录 ${ }^{[43]}$. 这种苯胺的光化学氧化只有在获得对称的 偶氮苯是有用的. 当等物质的量的两个不同胺的混合物 在 $\mathrm{HgO}$ 存在的条件下, 被光照射, 形成两个堆成的和一 个不对称的偶氮苯, 他们的产率适中或偏低. 作者解释 这种结果是因为在光照射的过程中, 不同过渡基团耦合 就已形成.

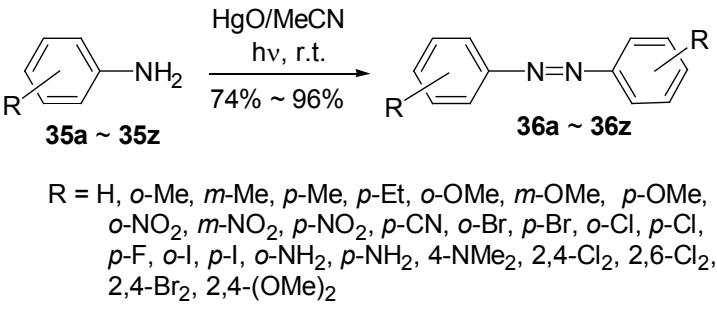

2006 年 $\mathrm{Chi}$ 等 ${ }^{[33]}$ 报道用 $\mathrm{MnO}_{2}$ 在苯溶液中氧化对苯 二胺衍生物 37 得到 4,4'-二氨基偶氮苯衍生物 38, 进而 衍生化制备了其他的 4,4'-二氨基偶氮苯衍生物 39, 40 (Scheme 5). 2007 年 Schmitzer 等 ${ }^{[44]}$ 报道用 $\mathrm{KO}_{2}$ 在 $\mathrm{THF}$ 中氧化对苯二胺 37 得到 4,4'-二氨基偶氮苯(38), 进而衍 生化制备了高分子偶联剂 39 (Scheme 5). 2009 年, Sadovski 等 ${ }^{[45]}$ 报道用 $\mathrm{AgO}$ 在丙酮中氧化单乙酰对苯二 胺的取代衍生物 40a 40f 得到 4,4'-二乙酰氨基偶氮苯 的取代衍生物 41a 41f (Scheme 6), 进而把它们衍生化 制备了光开关型偶联剂. 这些方法的缺点是过量使用金 属氧化物，反应后生成大量的金属盐，特别是过渡金属 或重金属的盐类. 因此，它们都不是环境友好型氧化剂. 迄今为止，很少有例子描述这种反应的催化进程.



\section{Scheme 5}

2008 年 Grirrane 等 ${ }^{[46]}$ 报道了以氧为氧化剂, 二氧化 钛承载的金纳米粒催化取代苯胺 $42 \mathrm{a} \sim \mathbf{4 2 f}$ 氧化生成芳 香偶氮化合物 $43 a \sim 43 f$, 且具有较高的选择性和好的产 
率(Scheme 6). 他们还研究了从硝基苯通过一锅法两步 合成偶氮苯. 首先, 在 $\mathrm{Au} / \mathrm{TiO}_{2}$ 上用氢还原硝基苯衍生 物, 然后, 用同样的催化剂氧化所得到的苯胺衍生物 44a 44f, 得到较高产率的偶氮苯衍生物 $45 \mathrm{a} \sim 45 \mathrm{f}$ (Eq. 7). 张等 ${ }^{[47]}$ 描述了利用空气或者分子氧作为氧化剂和铜 作为催化剂在温和的条件下由苯胺衍生物 $46 a \sim 46 f$ 合 成偶氮化合物 $47 \mathbf{a} \sim 47 \mathrm{f}$ (Eq. 8). 与吡啶鳌合的铜(I)被氧 气氧化以形成活性更强的复合物, 苯胺单电子氧化生成 相应的自由基正离子, 随后与另一分子的苯胺结合形成 一个含有三电子 $\sigma$ 键的中间体, 这种物质是鲍林 1931 年提出来的. 含有三电子 $\sigma$ 键的中间体捐出两个质子和 一个电子后, 形成联胺. 形成的联胺随后被铜(II)络合 物或者氧气氧化产生相应的偶氮化合物.<smiles>Nc1ccc(N=Nc2ccc(N)cc2)cc1</smiles>

42

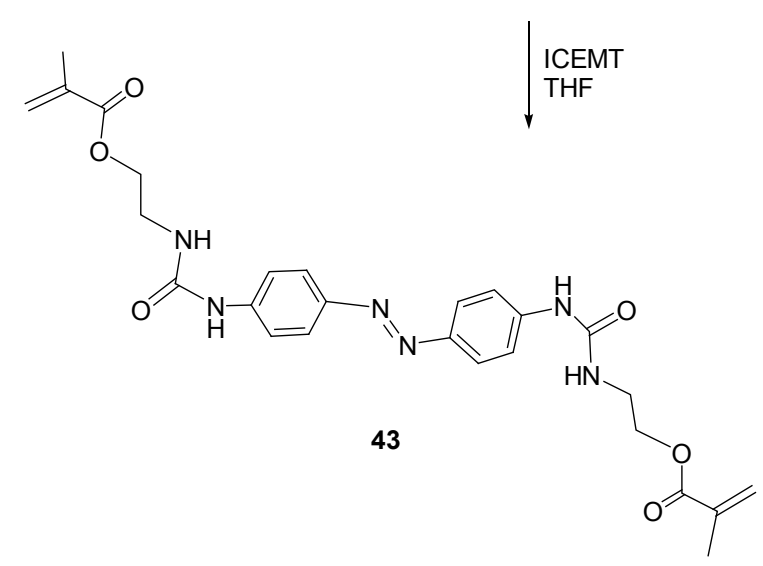

Scheme 6

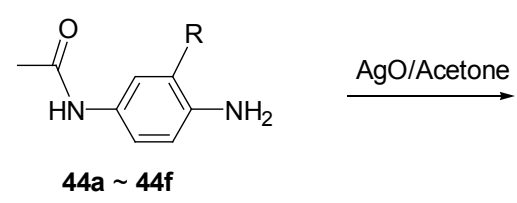<smiles>[R]c1cc(NC(C)=O)ccc1N=Nc1ccc(NC(C)=O)cc1[R]</smiles>

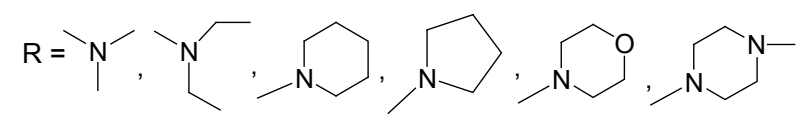

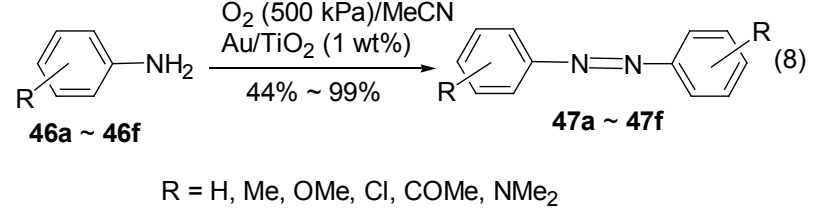

次卤酸钠氧化芳胺生成对称芳香偶氮化合物也是 近来发展的方便而有效的合成方法之一. Shaieb 等 ${ }^{[48]}$ 采 用次氯酸钠在碱性条件 $(\mathrm{KOH}$ 溶于 $95 \%$ 的酒精中)下低 温 $\left(-8{ }^{\circ} \mathrm{C}\right)$ 反应 $0.5 \mathrm{~h}$ 左右, 成功实现了把分子内的两个 芳基胺 48 氧化偶联成为相应的偶氮化合物 49, 产率为 $96 \%$ (Eq. 9). 之后, Woolley 等 ${ }^{[49,50]}$ 用次氯酸钠溶液在温 和的条件下直接把水溶性的芳胺衍生物 $\mathbf{5 0 , 5 6}$ 氧化成为 相应的对称型芳香偶氮化合物 51, 57 (Scheme 7), 并进 一步把它们衍生化制备光开关型分子交联剂(Scheme $8)^{[51,52]}$. 特别是最近, Minakata 等 ${ }^{[33]}$ 发现在碘化钠的催化 下，叔丁基氧氯能够有效地氧化芳基胺类化合物 59, 64 生成相应的对称型芳香偶氮化合物 $\mathbf{6 0}, \mathbf{6 6}$, 也能够把芳 基胺类化合物 61, 64 和芳基胺类化合物 62, 61a 组合氧 化生成不对称型芳香偶氮化合物 63, 65 (Scheme 9). 他 们还系统地详细讨论了各种反应条件如反应溶剂(如乙 腈、乙醚、丙酮、四氢呋喃和二甲氧基乙烷等)、反应 温度 $\left(-20 \sim 0{ }^{\circ} \mathrm{C}\right.$ 和常温)、反应时间 $(1,3,6,12,24 \mathrm{~h})$ 等 对生成物产率的影响, 并提出了该反应的机理.

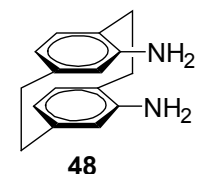

48
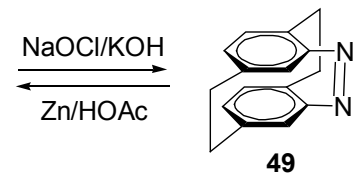

Renner 等 $\left.{ }^{[54}\right]$ 报道对苯二胺 $\mathbf{6 7}$ 的氨基在 3\%氢氧化 钠的碱溶液中加热到 $95{ }^{\circ} \mathrm{C}$, 可以被对硝基苯甲酸 68 的 硝基氧化, 偶联生成芳香偶氮氨基酸类化合物 69, 以并 69 为原料成功合成了一系列的光开关型环肽, 用于研 究光对环肽构象的调控变化情况. 此外, 李玉川等 ${ }^{[54 b]}$ 用二氯异氧尿酸钠(SDCI)氧化偶联 1-氨基-1,2,3-三唑高 收率合成了 8 个氮原子直接相连的 $1,1^{\prime}$-偶氮- $1,2,3$-三唑. 亚硝基芳香衍生物与苯胺在冰醋酸中可发生米尔斯 (Mills)反应生成相应的芳香偶氮化合物, 产率较高. 其 中的亚硝基芳香衍生物可以从相应芳胺的氧化反应(如 臭氧或 $2 \mathrm{KHSO}_{5} \cdot \mathrm{KHSO}_{4} \cdot \mathrm{K}_{2} \mathrm{SO}_{4}$ 在 $\mathrm{H}_{2} \mathrm{O} / \mathrm{CH}_{2} \mathrm{Cl}_{2}$ 中的非均 相体系氧化)或硝基芳香化合物的还原反应来制 备 ${ }^{[55-58]} .2010$ 年, Herges 等 ${ }^{[59]}$ 先用金属锌粉在碱性条件 下把芳香硝基化合物还原成为相应的亚硝基化合物 71, 再用得到的亚硝基化合物 71 氧化取代芳胺 70 制备了一 系列香偶氮化合物 72a $7 \mathbf{7 2} \mathbf{j}$ (Scheme 10). 
<smiles>CC(=O)Nc1ccc(NC(C)=O)c(S(=O)(=O)O)c1</smiles>

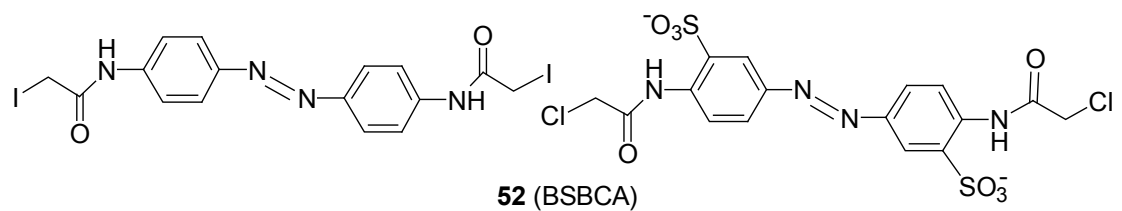

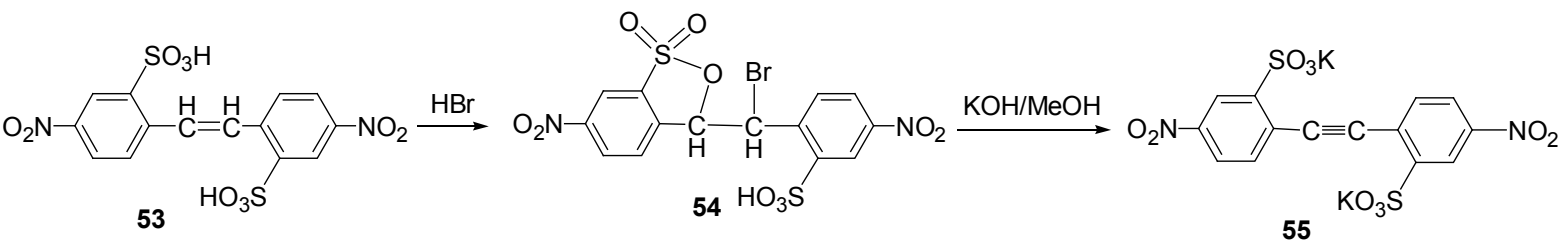<smiles></smiles>

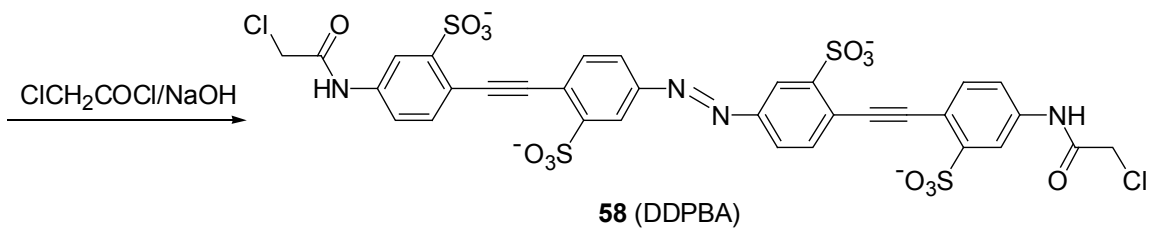

Scheme 7

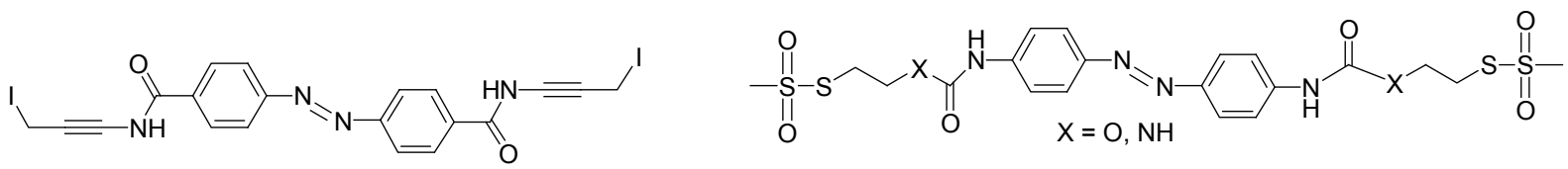<smiles>NCC#Cc1ccc(C(=O)NCc2ccc(N=Nc3ccc(C(=O)NCC#Cc4ccc(C(=O)O)cc4)cc3)cc2)cc1</smiles><smiles>CN(C#Cc1ccc(/N=N/c2ccc(/C=C/N(C)C(=O)CCl)cc2)cc1)C(=O)CCl</smiles><smiles>O=C(CCl)Nc1ccc(N=Nc2ccc(-c3ccc(N=Nc4ccc(NC(=O)CCl)cc4)cc3[S+](=O)[O-])c([S+](=O)[O-])c2)cc1</smiles>

BPDBS

Scheme 8 


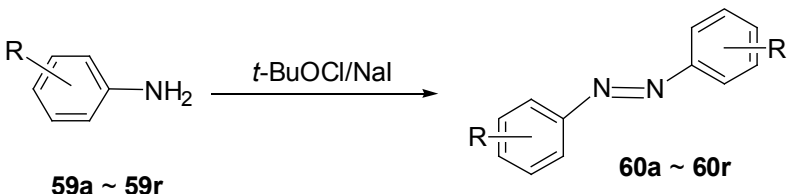

$\mathrm{R}=\mathrm{H}, p-\mathrm{Me}, p-\mathrm{OMe}, p-\mathrm{F}, p-\mathrm{Cl}, p-\mathrm{Br}, p-\mathrm{I}, p-\mathrm{CO}_{2} \mathrm{Et}, p-\mathrm{C}(\mathrm{O}) \mathrm{Et}, p-\mathrm{CN}, p-\mathrm{NO}_{2}, m-\mathrm{Cl}, m-\mathrm{NO}_{2}, o-\mathrm{Ph}, o-\mathrm{CN}, 3,4-\mathrm{Me}_{2}, 3,5-\left(\mathrm{CF}_{3}\right)_{2}, 2,3,4,5,6-\mathrm{F}_{5}$

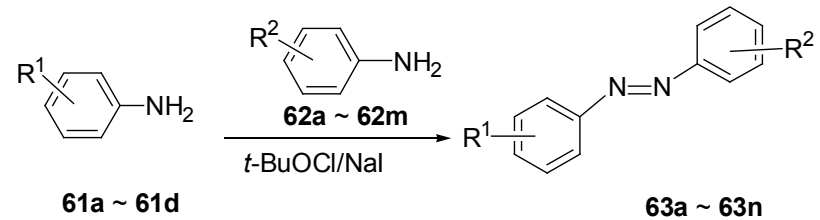

$\mathrm{R}^{1}=p$-Me, $p$-OMe, $p-\mathrm{CO}_{2} \mathrm{Et}, p-\mathrm{C}(\mathrm{O}) \mathrm{Et}$

$\mathrm{R}^{2}=\mathrm{H}, p-\mathrm{OMe}, p-\mathrm{F}, p-\mathrm{Cl}, p-\mathrm{Br}, p-\mathrm{I}, p-\mathrm{CO}_{2} \mathrm{Et}, p-\mathrm{C}(\mathrm{O}) \mathrm{Et}, p-\mathrm{NO}_{2}, m-\mathrm{Cl}, m-\mathrm{NO}_{2}, 3,5-\left(\mathrm{CF}_{3}\right)_{2}$

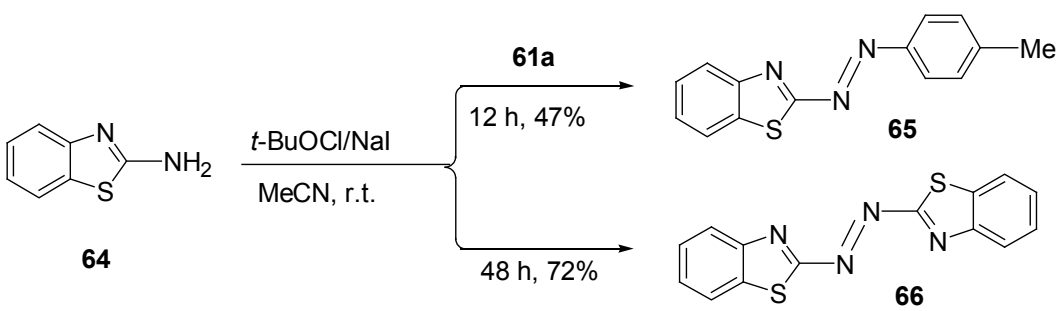

Scheme 9

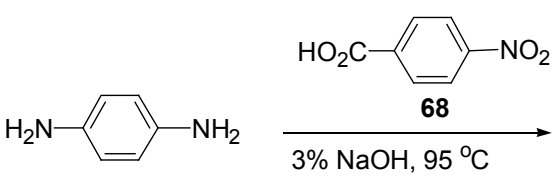

67<smiles>Nc1ccc(/N=N/c2ccc(C(=O)O)cc2)cc1</smiles><smiles>[R]c1ccc(/N=N/c2ccc([R])cc2)cc1</smiles>

$\mathrm{R}^{1}=\left(\mathrm{H}_{3} \mathrm{C}\right)_{3} \mathrm{Si}=$ or Fmoc-N-CH

$\mathrm{R}^{2}=\mathrm{H}, p-\mathrm{I}, \quad p-\mathrm{CN}, p-\mathrm{Me}, \quad p-\mathrm{CF}_{3}, p-n-\mathrm{Hex}, p-\mathrm{CO}_{2} t-\mathrm{Bu}$,

$3,5-\mathrm{Me}_{2}, 3,5-t-(\mathrm{Bu})_{2}$

\section{Scheme 10}

\section{3 硝基芳香化合物的还原偶联反应}

硝基苯甲酸盐与还原剂 $\mathrm{NaBH}_{4}, \mathrm{LiAlH}_{4}$ ，乙二醇中 的 2-羟基乙醇钠, $\mathrm{KOH}^{[60]}, \mathrm{Zn} / \mathrm{NaOH}^{[61]}, \mathrm{Bi}^{[62]}, \mathrm{Bi}-$ $\mathrm{KOH}^{[63]}$ 或者 $\mathrm{Pb} / \mathrm{HCO}_{2} \mathrm{NH}_{4}{ }^{[64]}$ 等的还原偶联是一种获得 独特对称结构的芳香偶氮化合物的有效方法. 在甲醇溶 液中和室温条件下, 用 $\mathrm{Mg} / \mathrm{HCO}_{2} \mathrm{HNEt}_{3}$ 与 2-硝基甲苯
(73)还原偶联，以 $90 \%$ 的产率生成 2,2'二甲基偶氮苯(74) (Eq. 10) $)^{[65]}$.

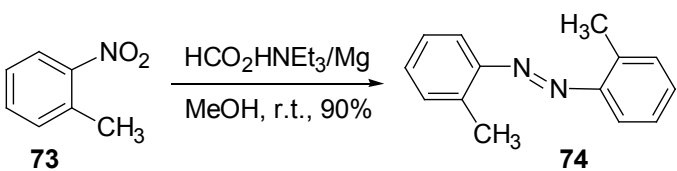

其他还原剂已经被用于硝基芳烃的还原偶联，产生 相应的偶氮苯的产率从 $60 \% \sim 95 \%$ ，这些还原剂包括超 声条件下 $\mathrm{Al} / \mathrm{NaOH}^{[66]}, \mathrm{SnCl}_{2} / \mathrm{NaOH}^{[67]}$ ，葡萄糖/ $\mathrm{NaOH}^{[68]}$ (Scheme 11), $\mathrm{Pb} / \mathrm{HCO}_{2} \mathrm{HNEt}_{3}{ }^{[69]}, \mathrm{Pb} / \mathrm{CH}_{3} \mathrm{CO}_{2} \mathrm{NH}_{4}{ }^{[70]}$,<smiles>O=C(O)c1ccc(N=Nc2ccc(C(=O)O)cc2)cc1</smiles>

75
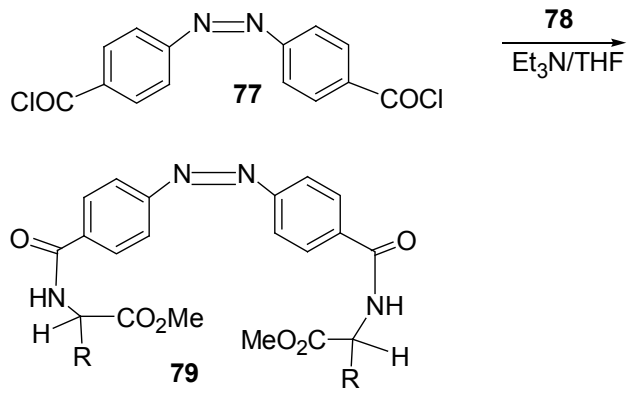

$78 \mathrm{RCH}\left(\mathrm{NH}_{2}\right) \mathrm{CO}_{2} \mathrm{Me}$

$\mathrm{R}=i-\mathrm{Pr}, \mathrm{Me}, i-\mathrm{Bu}, \mathrm{Bz}$, Melndo, $p-\mathrm{OH}-\mathrm{Bz}, \mathrm{CH}_{2} \mathrm{OH}$

Scheme 11 
$\mathrm{TiCl}_{4} / \mathrm{LiAlH}_{4}, \mathrm{NaBH}_{4} /(\mathrm{PhTe})_{2}$ 或 $\mathrm{NaBH}_{4} / \mathrm{Pd}-\mathrm{C}$ (Eq. 11) ${ }^{[71]}$, $\mathrm{Co}_{2}(\mathrm{CO})_{8}$ 或 $\mathrm{NiCl}_{2}-\mathrm{H}_{2} \mathrm{O}-\mathrm{Li}-4,4{ }^{\prime}-$ 二叔丁基联苯(DTBB). 而且, 镁/二异丙胺, $\mathrm{FeO}$, 五羰基铁/ CO 或 $\mathrm{MCl}_{n}-\mathrm{Mg}$ THF 系统(这里 $\mathrm{MCl}_{n}=\mathrm{TiCl}_{4}, \mathrm{VCl}_{3}, \mathrm{CrCl}_{3}, \mathrm{MoOCl}_{3}$, $\mathrm{WCl}_{6}, \mathrm{FeCl}_{3}$ ) 也能用于这种目的.

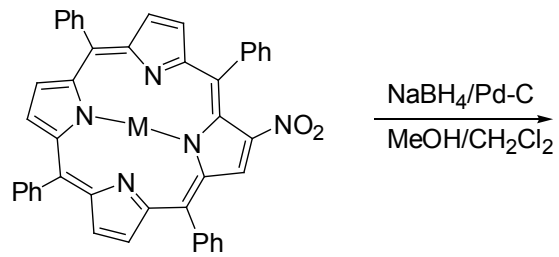

80

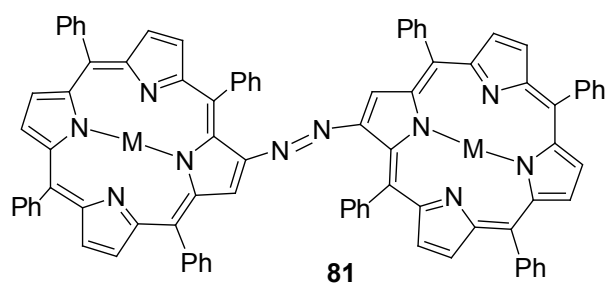

采用镁电极的电化学还原也能以较高的产率制备 偶氮化合物. 提出的这种还原偶联的可能机制是在酸或 碱存在时硝基化合物的还原 ${ }^{[72]}$. 因此, 硝基芳香化合物 先与还原剂反应生成亚硝基衍生物和相应的差胺的混 合物. 两者随后转变成自由基负离子, 结合产生 1 个 $\mathrm{N}-\mathrm{N}$ 键, 从而生成 $N, N^{\prime}$-二羟基中间体. 这种中间体脱 水形成氮化偶氮化合物是单限速步骤. 质子和氢氧根负 离子在酸性和碱性介质中消去的形式可能是经由水分 子. 最近, Sakai 及其同事 ${ }^{[73]}$ 报道了利用 $\mathrm{In}(\mathrm{OTf})_{3} / \mathrm{Et}_{3} \mathrm{SiH}$ 系统从硝基衍生物 82 获得偶氮苯 83 的产率达到 $62 \%$ 99\% (Eq. 12). 这种方法的一个缺点是还原剂使用过量, 还原剂形成的中间产物对环境不利.

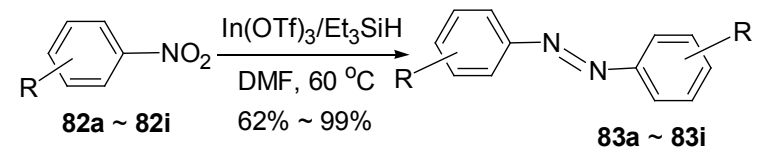

$\mathrm{R}=\mathrm{H}, o-\mathrm{Me}, m-\mathrm{Me}, p-\mathrm{Me}, p-\mathrm{Cl}, p-\mathrm{Br}, p-\mathrm{CN}, p-\mathrm{MeCO}, m-\mathrm{CO}_{2} \mathrm{Me}$

通过光催化过程还原硝基芳香化合物获得偶氮苯 的方法仅见一个的例子, $\mathrm{Zhu}$ 等 ${ }^{[74]}$ 最近报道了在 $\mathrm{ZrO}_{2}$ 支 撑的金纳米粒上，通过可见光或紫外线照射，催化硝基 苯衍生物 84 的还原. 在室温和常压下, 获得了高选择性 的偶氮化合物 85 (Eq. 13).

\section{4 芳香偶氮氧化物的转化与还原}

经典的瓦拉赫(Wallach) 反应涉及到氧化偶氮芳香 化合物经酸处理后转变成 4-差基取代芳香偶氮衍生物 (或有时 2-羟基异构体). 氧化偶氮苯可以通过硝基苯与

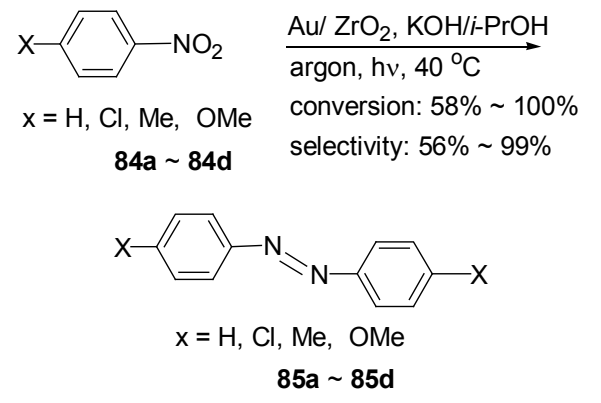

酒精氢氧化钾/乙醛、钠录齐、氢 $/ \mathrm{PbO}$ 或 $\mathrm{MeONa}$ 还原而轻易获得, 或者经氧化苯胺与过氧化氢或高锰酸 /甲醛. 如所示，4-硝基衍生物 86a 在硫酸溶液中加热到 $100{ }^{\circ} \mathrm{C}$ 反应，生成产物以 2-羊基偶氮苯衍生物 87a 为主 [75]. 而当取代基 $\mathrm{X}$ 为乙酰基或羧基时，同样的反应条件 下则分别获得 IPSO 重排产物 $88 b$ 或 88c (Eq. 14).

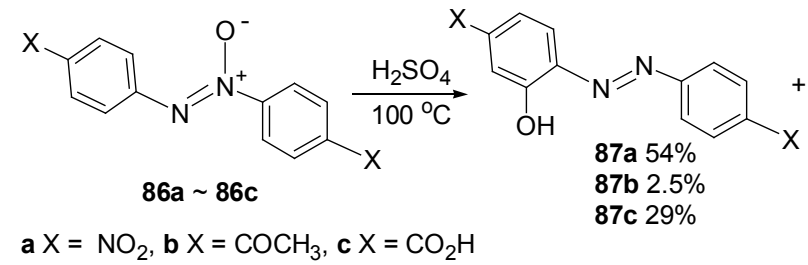<smiles>[X]c1ccc(N=Nc2ccc(O)cc2)cc1</smiles>

对芳香偶氮氧化物 90 和路易斯酸的研究表明, 90 与五氯化锑反应以高产率地形成了 $1: 1$ 的复合物 91 . 然而, 它们具有高吸湿性, 并且它们的水解会引发芳香 偶氮氧化物启动热分解反应. 90 与 $\mathrm{SbCl}_{5}$ 形成的复合物 91 在硝基苯中的热解可选择性的生成 2-羟基取代的产 物 92, 产率可达 7\% 73\%，同时也可能形成焦油状产 物. 产物 92 与焦油状产物的分配具有高度的温度依赖 性. 例如, 对于氧化偶氮苯, 焦油状产物随温度的增加 而增加, 在 $85{ }^{\circ} \mathrm{C}$ 时获得偶氮苯的最大产率 $($ Scheme $12)^{[76]}$. 使用其他路易斯酸, 如四氯化钛、三氯化铝、三 氯化铁和二溴化锌，通过邻位拉瓦赫热反应反应形成的 复合物没有发生. 氧化偶氮苯过量使用路易斯酸时，获 得对位氯偶氮苯和偶氮苯的混合物. 主要反应是脱氧以 生成偶氮苯. 另外，二硫化碳或醋酰氯中，氧化偶氮苯 93 与三氯化铝或三氯化铁热反应主要生, 成 4-氯偶氮 苯97 并有痕量的 2-羟基苯. 从反应机理的观点来看，假 设路易斯酸最初的与氧化偶氮基上的氧结合而形成配 合物 94, 给通过共振得以稳定的物质让出位置，例如 

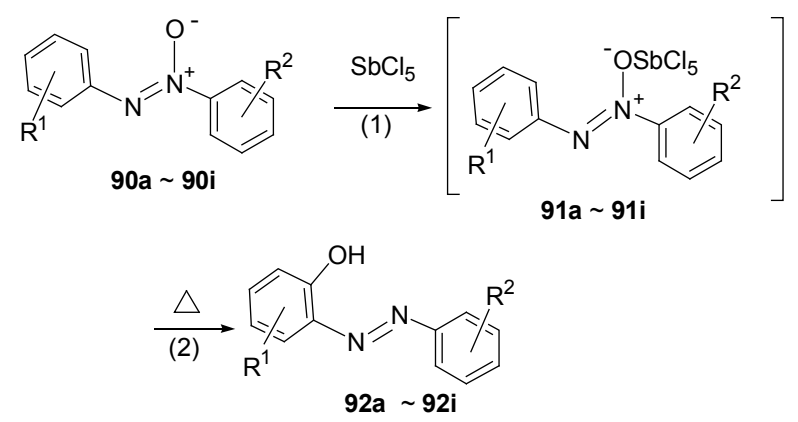

$\mathrm{R}^{1}=\mathrm{H}, \mathrm{o}-\mathrm{Me}, \mathrm{o}-\mathrm{Me}, m-\mathrm{Me}, p-\mathrm{Me}, \mathrm{H}, \mathrm{H}, \mathrm{H}, \mathrm{H}$

$\mathrm{R}^{2}=\mathrm{H}, \mathrm{H}, o-\mathrm{Me}, m-\mathrm{Me}, \mathrm{H}, p-\mathrm{Me}, p-\mathrm{Cl}, p-\mathrm{Br}, p-\mathrm{NO}_{2}$

\begin{tabular}{cccccccccc}
\hline \multirow{2}{*}{ Route } & \multicolumn{10}{c}{ Yield/\% } \\
\cline { 2 - 11 } & a & b & c & d & e & f & g & h & i \\
\hline$(1)$ & 94 & 97 & 98 & 98 & 99 & 97 & 93 & 94 & 91 \\
$(2)$ & 64 & 34 & 13 & 16 & 73 & 32 & 12 & 47 & 7 \\
\hline
\end{tabular}

Scheme 12

95. 缺电子的中间体对位随后被氯负离子进攻形成 96, 去质子化后生成最终产物 97 (Scheme 13) ${ }^{[77]}$. 当把氧化 偶氮苯加热到 $245 \sim 250{ }^{\circ} \mathrm{C}$ 的温度时, 瓦拉赫热反应在 合成中是没有用的, 因为 2-羟基偶氮苯、2-羟基氧化偶 氮苯、4-差基偶氮苯和 4-羊基氧化偶氮苯的混合物的产 率较低. 采用 $\mathrm{H}_{2} \mathrm{O}_{2} / \mathrm{Na}_{2} \mathrm{WO}_{4}$ 时, 苯胺(98)先被氧化生成 偶氮二氧化物 99 , 它随后与 $\mathrm{Si}_{2} \mathrm{Cl}_{6}$ 发生还原反应, 以很 好的产率获得了偶氮苯 101 (Scheme 14) ${ }^{[78]}$.<smiles>CO[N+](=Nc1ccccc1)c1ccc(C(C)(C)C)cc1</smiles><smiles>CON(N=C1C=CCCC1C)c1ccccc1</smiles><smiles>CON(N=C1C=CC(Cl)C=C1)c1ccccc1</smiles>

Scheme 13

偶氮苯也可以通过氮化偶氮基衍生物直接还原的 方法制备得到. 最近报道了在金属铝或锡存在的情况 下, 用水合肼处理氧偶氮基芳烃 $\mathbf{1 0 2}$, 在甲醇中回流或

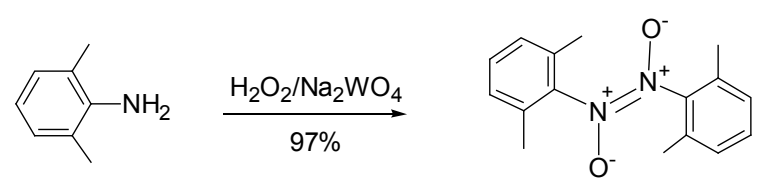

98

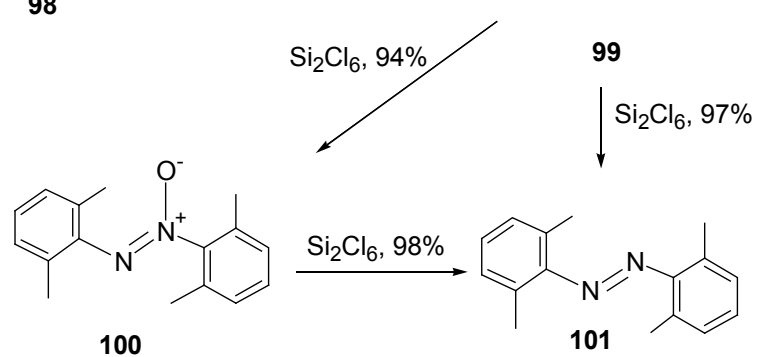

Scheme 14

用微波辐射, 反应速度很快并以极好的产率获得偶氮芳 烃 103 (Eq. 15) ${ }^{[79,80]}$. 利用 $\mathrm{AlI}_{3}$ 或者 $\mathrm{InCl}_{3}$ 和金属的三氟 甲磺酸盐，例如 $\mathrm{Zn}(\mathrm{OTf})_{2}$ 或 $\mathrm{Cu}(\mathrm{OTf})_{2}$ 也能以极好的产 率生成偶氮化合物 ${ }^{[81 ~ 83]}$. 在乙醇和水中加入含有酵母 的碱性还原溶液, 对于快速和选择性还原氮化偶氮苯化 合物是一种很有效的方法 ${ }^{[84]}$. 当反应被二氯二氧化锄 催化时, 三级磷化氢类也可以被用作还原剂 ${ }^{[85]}$. 被碘和 三亚磷酸酯类激活的三(二甲胺基)磷化氢也可以以较好 的产率从氧化偶氮苯生成偶氮化合物 ${ }^{[66,87]}$.
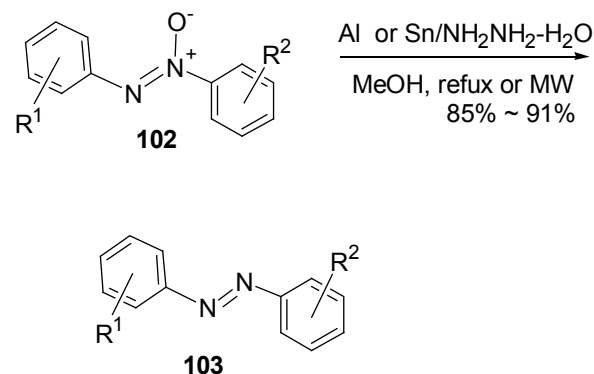

$\mathrm{R}^{1}, \mathrm{R}^{2}=\mathrm{H}, o$-Me, $m$-Me, $p$-Me, o-OMe, $p$-OMe, o-OEt, o-Cl, $m-\mathrm{Cl}, p-\mathrm{Cl}$

\section{5 叠氮芳香化合物的催化偶联与热分解反应}

在苯胺存在时, 加热叠氮芳香化合物将产生低产率 的不对称偶氮化合物. 例如, 当 4-硝基叠氮苯 $104(\mathrm{X}=$ $\left.p-\mathrm{NO}_{2}\right)$ 和 4-甲基苯胺 $105(\mathrm{Y}=p-\mathrm{Me})$ 一起共热到 $135{ }^{\circ} \mathrm{C}$ 的时候, 分解生成取代偶氮苯 106, 产率为 $16 \%$ (Eq. $16)^{[88]}$. 当两个环上取代基 $(\mathrm{X}$ 和 $\mathrm{Y})$ 的电子效应相反时, 这种反应更有效. 叠氮化合物中取代基 $\mathrm{X}$ 的结构特性比 苯胺中的 $\mathrm{Y}$ 更关键. 由于叠氮化合物易爆且难以处理, 这种方法的实用性是非常有限的. Peters 等 ${ }^{[89,90]}$ 最近报 道了一种叠氮芳香化合物 107 直接催化偶联生成芳香偶 氮化合物 108 的方法(Eq. 17), 与叠氮芳香化合物的热分 解反应相比, 这种方法的优点在于反应温度较低, 转化 率较高, 而且不需要芳基胺参与反应, 产物更易于纯化. 
<smiles>[X]c1ccc(N=Nc2cccc(N=Nc3cccc([Y])c3)c2[X])cc1</smiles><smiles>[Z]c1cccc(N=Nc2cccc(N=Nc3ccccc3[Z])c2[Z])c1</smiles>

107

$$
\text { 催化剂: NAr }
$$

四苯基卟啉钴

A

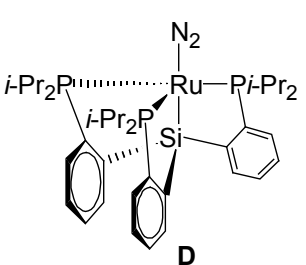

$\left[\mathrm{SiP}(i-\mathrm{Pr})_{3}\right] \mathrm{Fe}(\mathrm{NAr})$

E

\section{6 芳香基重氮盐的偶合与催化偶联反应}

大多数的偶氮苯是通过这种类型的反应获得的. 方 法是建立在低温下芳香伯胺的初期重氮化作用, 接着它
又与一个富电子芳香化合物的亲电取代反应，反应时间 短, 产量高. 例如, 在 $0{ }^{\circ} \mathrm{C}$ 下利用 $\mathrm{K}_{2} \mathrm{CO}_{3}$ 作为碱, 使对 硝基苯重氮盐和邻甲氧基苯酚反应合成偶氮化合物的 产率达到 $92 \%{ }^{[91]}$. 重氮盐是弱亲电性的物质, 它与富电 子物质反应，例如像胺基和羟基等取代的芳烃具有给电 子集团，供给偶氮苯。一般说来，这种取代反应通常是 在较活泼的芳环上发生在供电子基团的对位上. 当这个 位置已经被占据，取代发生在邻位. 芳胺 $(109,113,116$, 118)在低温下经亚硝酸氧化生成重氮盐(110, 114), 再与 酚类化合物在弱碱性条件下发生偶合反应得到芳香偶 氮衍生物 $(112,115,117,119)$, 实例如 Scheme 15 所 示 ${ }^{[92 \sim 95]}$

这类反应具有较强的 $\mathrm{pH}$ 依赖性. 关于起始原料的 反应性, 酚类必须以电离的形式经历偶联反应, 因为中 性物质亲核性不足. 在这种情况下，温和的碱性溶液时 必要的, 因为重氮盐在碱存在的情况下变成重氮氢氧化 物，从而抑制偶联. 因此，酚类的偶联必须在控制 $\mathrm{pH}>$ 7 的弱碱介质中进行. 然而, 芳香胺应该在弱酸介质中 进行，以防止胺基的 $\mathrm{N}$-偶合，因为介质呈弱碱性时胺基 中氮的亲核性并不减弱，而如果在强酸中，它将被质子 化. 芳基重氮盐与芳胺类化合物偶合生成芳香偶氮化合 物的应用实例如 Scheme 16 所示 ${ }^{[52,96]}$.
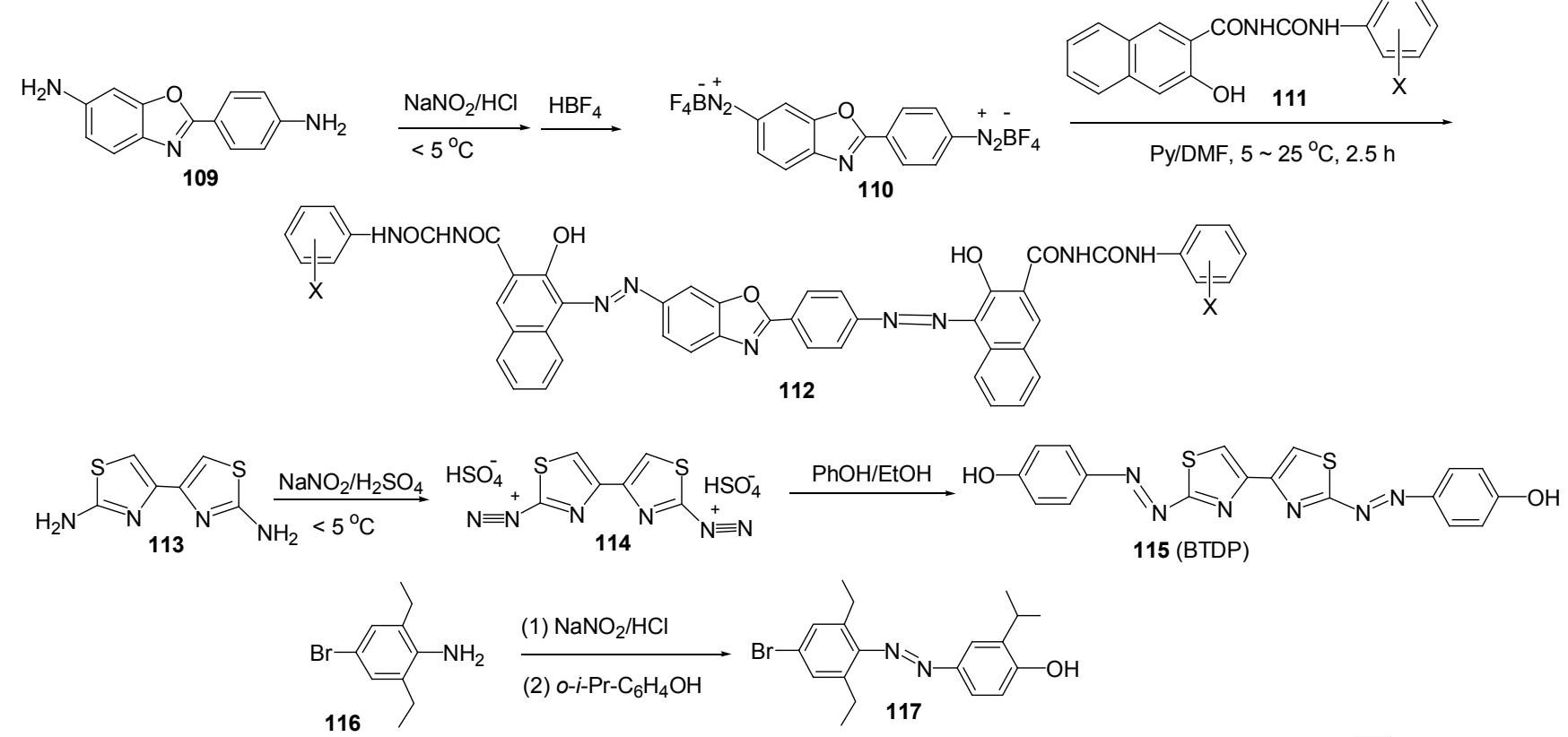<smiles>Nc1ccc2c(c1)Cc1cc(N)cc(N)c1-2</smiles>

118

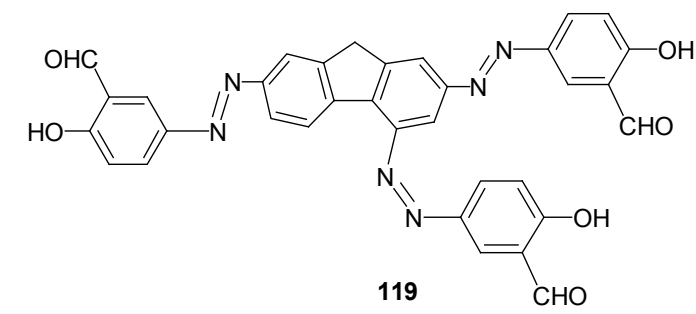

Scheme 15 


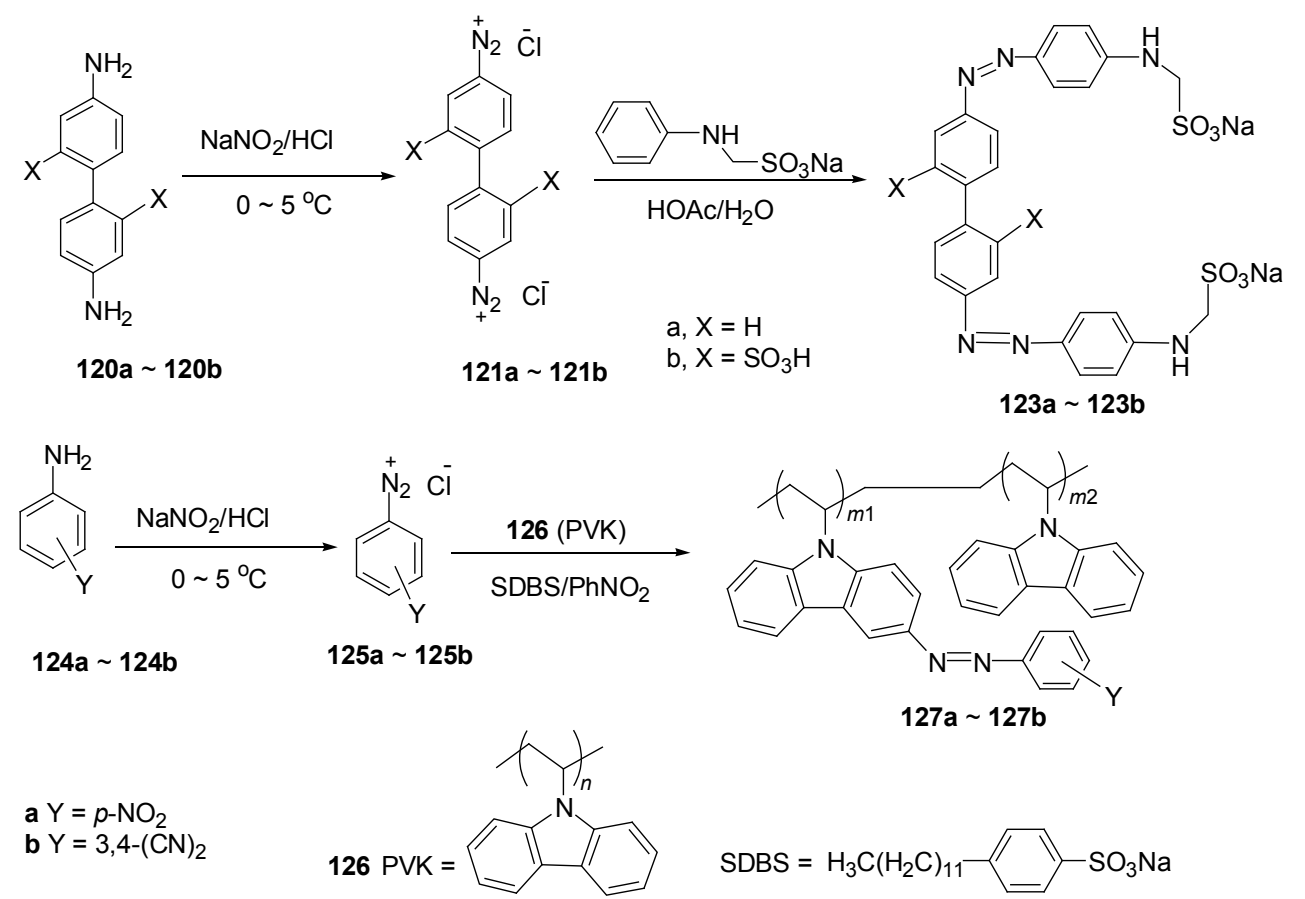

Scheme 16

重氮盐的偶联反应也可以在固相中发生. 在这个进 程中, 重氮盐在聚苯乙烯树脂(大孔树脂 A-15, 磺酸基 团树脂和季铵阳离子功能化大孔树脂 A-26)上与磺酸盐 基经历阴离子交换. 通过这种交换产生的重氮盐 128 在 多聚支撑物上与富电子的酚类芳香族化合物 130 偶联形 成偶氮化合物 131, 不经繁琐的处理和分离步骤就可以 纯化 (Scheme 17) ${ }^{[97]}$. 此外, 偶氮偶联已经允许在卟啉 类、间环芳和杯芳烃 ${ }^{[98]}$ 中引入偶氮基团.<smiles>Nc1ccc(Br)cc1</smiles>

128

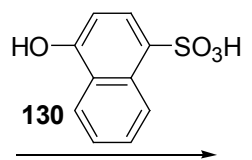

$\mathrm{NH}_{3}-\mathrm{H}_{2} \mathrm{O}$
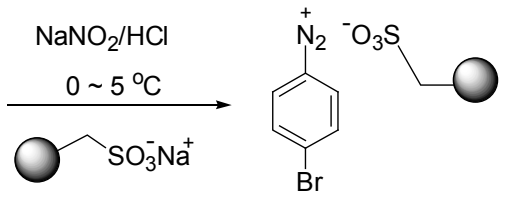

129

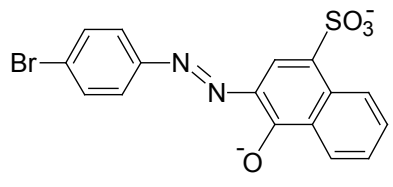

131
Scheme 17

重氮盐的平衡反离子特性也强烈地影响到它的稳 定性. 氯化物是不稳定的, 它们在 $5{ }^{\circ} \mathrm{C}$ 以上剧烈分解. 四氟硼酸盐、二磺酰胺、六氟磷酸盐和氯化锌在固体形 式下是稳定的, 可以长时间保存. 这些盐容易与有机金 属试剂反应，通过简单添加有机金属到含重氮基的溶液 中即能反应. 人们已经研究发现重氮-2-苯磺酰胺等重
氮盐类在格林试剂 ${ }^{[99]}$, 镉、录、锂、锌和锡等金属及其 有机化合物的催化作用下可以发生自偶聚反应生成相 应的芳香偶氮衍生物. 特别是, 在苯基重氮四氟硼酸盐 (133) 与二苯基锌之间的反应中获得了较高产率 $(72 \%$ 95\%)的偶氮苯及其衍生物 134 (Scheme 18). 除此之外, 还有其他一些反应可以应用于合成芳香偶氮化合物, 如 三氮烯的重排反应、 $N, N^{\prime}$-对苯醌二亚胺二氧化物的分解 反应、芳香钻衍生物与氧化亚氮的反应以及苯并三唑的 开环反应等 ${ }^{[100]}$.

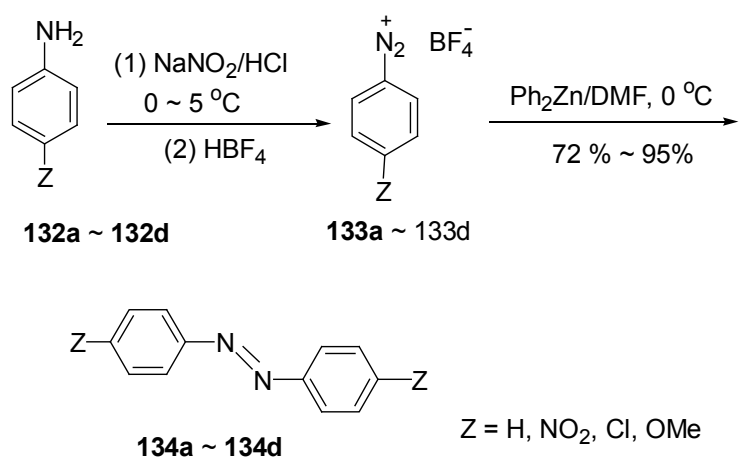

Scheme 18

\section{2 总结与展望}

由于芳香偶氮衍生物性质广泛，应用较广，所以它 是非常重要的化合物. 本文综述总结了当前文献中报道 的新芳香偶氮衍生物的合成方法及其应用进展. 通常最 有用的方法是偶氮偶联, 米尔斯反应和瓦拉赫反应，但 
针对某些特定对称型芳香偶氮衍生物的合成而言，这些 方法存在一些不足或不便之处. 除此之外, 偶氮偶联反 应中, 使用的重氮盐可能爆炸, 而且必须控制反应的温 度. 米尔斯和瓦拉赫合成亚硝基化合物和偶氮苯的反应 中, 必须使用化学计量或者过量的氧化剂和还原剂且同 时生成较多的副产品, 给目标产物的分离纯化带来麻 烦. 在氧化偶氮苯的还原中, 仍然需要使用过量的还原 剂. 最近, 因为对称芳香偶氮衍生物在光开关分子化 学、主客体识别、超分子化学、自组装液晶材料、生物 医学成像与化学分析以及光驱分子马达等诸多领域的 应用潜力更大, 使得通过芳香硝基衍生物还原偶联、氧 化苯胺及其衍生物和芳香肼脱氢等合成偶氮苯的方法 越来越受到重视, 但这些方法的催化策略的报道相对较 少. 苯并三坐的开环仅仅允许获得 2-羟-偶氮苯. 重氮盐 的二聚化作用, 三氮烯的重排, 叠氮化合物的热解, 氧 化亚氮与芳香钙衍生物的反应, $N, N^{\prime}$-对苯醌二亚胺二氧 化物的分解得到的偶氮化合物的产率较低且对这些制 备方法的兴趣受限. 通过金属催化芳基肼偶联合成的偶 氮苯产率较高. 醌类和苯醌缩醛与芳基肼的反应允许在 温和条件下合成偶氮化合物, 且产率较高. 虽然有不同 的获得偶氮苯的方法, 但是他们都表现出局限性, 有必 要继续研究以发现新的合成方法以适应新的需求, 特别 是更注重研发一些低毒高效又绿色环保的合成策略. 总 之, 光致异构的芳香偶氮衍生物不仅激发和丰富了化学 家的创造欲和想象力, 而且还为材料学家、生物学家和 药物学家都带来前所未有的创新机遇和开发空间.

\section{References}

[1] Cannizzo, C.; Amigoni-gerbier, S.; Frigoli, M.; Larpent C. J. Polym. Sci.: Part A: Polym. Chem. 2008, 6, 3375.

[2] Brenzovich, Jr., E. W.; Houk, J. T. R.; Malubay, M. A. S.; Miranda, O. J.; Ross, M. K.; Abelt, J. C. Dyes Pigm. 2002, 52, 101.

[3] Athey Jr., R. D. Eur. Coat. J. 1998, 3, 146.

[4] Sandborn, W. J. Am. J. Gastroenterol. 2002, 97, 2939.

[5] (a) Jain, A.; Gupta, Y.; Jain, S. K. Crit. Rev. Ther. Drug Carrier Syst. 2006, 23, 349.

(b) Mei, X.; Yang, S.; Chen, D. Y.; Li, N. J.; Li, H.; Xu, Q. F.; Ge, J. F.; Lu, J. M. Chem. Commun. 2012, 48, 9846.

[6] Cisnetti, F.; Ballardini, R.; Credi, A.; Gandolfi, M. T.; Masiero, S.; Negri, F.; Pieraccini, S.; Spada G. P. Chem.-Eur. J. 2004, 10, 2011.

[7] (a) Li, W. W.; Xu, D. M.; Zhang, Z. L.; Xu, Y. Chin. J. Chem. 2012, 30, 1819.

(b) Natansohn, A.; Rochon, P. Chem. Rev. 2002, 102, 4139.

[8] Lee, K. M.; Wang, H. D.; Koerner, H.; Vaia, A. R.; Tan, L.-S.; White J. T. Angew. Chem., Int. Ed. 2012, 51, 4117.

[9] Deloncle, R.; Caminade, A.-M. J. Photochem. Photobiol. C: Photochem. Rev. 2010, 11, 25.

[10] (a) Bandara, D.; Burdette, C. S. Chem. Soc. Rev. 2012, 41, 1809. (b) Ikeda, T.; Tsutsumi, O. Science 1995, 268, 1873.

[11] Tamaoki, N. Adv. Mater. 2001, 13, 1135.

[12] Feringa, B. L.; Van Delden, R. A.; Koumura, N.; Geertsema, E. M. Chem. Rev. 2000, 100, 1789.
[13] (a) Wegner, A. H. Angew. Chem., Int. Ed. 2012, 51, 4787.

(b) Beharry, A. A.; Woolley, G. A. Chem. Soc. Rev. 2011, 40, 4422.

(c) Hoppmann, C.; Seedorff, S.; Richter, A.; Fabian, H.; Schmieder, P. Angew. Chem., Int. Ed. 2009, 48, 6636.

(d) Gorostiza1, P.; Isacoff, Y. E. Science 2008, 322, 395.

[14] Beharry, A. A.; Wong, L.; Tropepe, V.; Woolley, G. A. Angew. Chem., Int. Ed. 2011, 50, 1325.

[15] Murakami, H.; Kawabuchi, A.; Kotoo, K.; Kutinake, M.; Nakashima, N. J. Am. Chem. Soc. 1997, 119, 7605.

[16] Banerjee, I. A.; Yu, L.; Matsui, H. J. Am. Chem. Soc. 2003, 125, 6542.

[17] Crano, J. C.; Guglielmetti, R. Organic Photochromic and Thermochromic Compounds, Plenum Press, New York, 1999.

[18] (a) Bandara, H. M. D.; Burdette, C. S. Chem. Soc. Rev. 2012, 41, 1809.

(b) Muraoka, T.; Kinbara, K.; Aida, T. Nature 2006, 440, 512.

[19] Liang, X.; Asanuma, H.; Komiyama, M. J. Am. Chem. Soc. 2002, 124, 1877.

[20] Zhen, W.; Han, H.; Anguiano, M.; Lemere, C.; Cho, C.-G.; Lansbury, P. T. J. Med. Chem. 1999, 42, 2805.

[21] Barth, M.; Tasadaque, S.; Shah, A.; Rademann, J. Tetrahedron 2004, 60, 8703.

[22] Samanta, S.; Qin, C. G.; Lough, J. A.; Woolley, G. A. Angew. Chem., Int. Ed. 2012, 51, 6452.

[23] Hyatt, J. A. Tetrahedron Lett. 1977, 18, 141.

[24] Drug, E.; Gozin, M. J. Am. Chem. Soc. 2007, 129, 13784.

[25] Niu, Y.-S.; Li, J.-P; Zheng, P.-Z. Chem. Reag. 2006, 28, 109 (in Chinese).

(牛永生, 李建平, 郑鹏志, 化学试剂, 2006, 28, 109.)

[26] Lim, Y.-K.; Lee, K.-S.; Cho, C.-G. Org. Lett. 2003, 5, 979.

[27] Kang, H. M.; Kim, H. Y.; Jung, J. W.; Cho, C. G. J. Org. Chem. 2007, 72, 679.

[28] Dohno, C.; Yamamoto, T.; Nakatani, K. Eur. J. Org. Chem. 2009, 4051.

[29] Kim, K. Y.; Shin, J. T.; Lee, K. S.; Cho, C. G. Tetrahedron Lett. 2004, $45,117$.

[30] Smith, L. I.; Irvine, W. B. J. Am. Chem. Soc. 1941, 63, 1036.

[31] Hecker, E.; Lattrell, R. Justus Liebigs Ann. Chem. 1963, 662, 48.

[32] Taylor, E. C.; Jagdmann, Jr. G. E.; McKillop, A. J. Org. Chem. 1978, 43, 4385.

[33] Naemura, K.; Wanebe, T.; Hirose, K.; Tobe, Y. Tetrahedron: Asymmetry 1997, 8, 873.

[34] Carreno, C. M.; Fernandez-Mudarra, G.; Merino, E.; Ribagorda, M. J. Org. Chem. 2004, 69, 3413.

[35] Carreno, C. M.; Garcia, I.; Ribagorda, M.; Merino, E.; Pieraccini, S.; Spada, G. P. Org. Lett. 2005, 7, 2869;

[36] Carreno, C. M.; Garcia, I.; Nunez, I.; Merino, E.; Ribagorda, M.; Pieraccini, S.; Spada, G. P. J. Am. Chem. Soc. 2007, 129, 7089.

[37] Schmittel, M.; Burghart, A. Angew. Chem., Int. Ed. 1997, 36, 2550.

[38] Patel, S.; Mishra, B. K. Tetrahedron Lett. 2004, 45, 1371.

[39] Noureldin, N. A.; Bellegarde, J. W. Synthesis 1999, 939.

[40] Karunakaran, C.; Palanisamy, P. N. J. Mol. Catal. A: Chem. 2001, $172,9$.

[41] Orito, K.; Hatakeyama, T.; Takeo, M.; Uchiito, S.; Tokuda, M.; Suginome, H. Tetrahedron 1998, 54, 8403.

[42] Farhadi, S.; Zaringhadam, P.; Sahamieh, R. Z. Acta Chim. Slov. 2007, 54, 647.

[43] (a) Chi, L.; Sadovski, O.; Woolley, G. A. Bioconjugate Chem. 2006, 17, 670 .

(b) Gilbert, M. A.; Failli, A.; Shumsky, J.; Yang, Y. J.; Severin, A.; Singh, G.; Hu, W. J. Med. Chem. 2006, 49, 6027.

(c) Norikane, Y.; Tamaoki, N. Org. Lett. 2004, 6(15), 2595. 
[44] Gomy, C.; Schmitzer, R. A. Org. Lett. 2007, 9(20), 3865.

[45] Sadovski, O.; Beharry, A. A. Zhang, F. Z.; Woolley, G. A. Angew. Chem., Int. Ed. 2009, 48, 1484.

[46] (a)Grirrane, A.; Corma, A.; Garcia, H. Science 2008, 322, 1661. (b) Esdaile, J. L.; Jensen, P.; McMurtrie, C. J.; Arnold, P. D. Angew. Chem., Int. Ed. 2007, 46, 2090.

(c) Leyva, E.; Medina, C.; Moctezuma, E.; Leyva, S. Can. J. Chem. 2004, 82, 1712.

[47] Zhang, C.; Jiao, N. Angew. Chem., Int. Ed. 2010, 49, 6174.

[48] Shaieb, E. K.; Narayanan, V.; Hopf, H. Eur. J. Org. Chem. 2003, 567.

[49] Burns, C. D.; Zhang, F. Z; Woolley, G. A. Nat. Protoc. 2007, 2, 251.

[50] Zhang, F. Z.; Sadovski, O.; Woolley, G. A. ChemBioChem 2008, 9, 2147.

[51] Beharry, A. A.; Sadovski, O.; Woolley, G. A. Org. Biomol. Chem. 2008, 6, 4323.

[52] Samanta, S.; Woolley G. A. ChemBioChem 2011, 12, 1712.

[53] Takeda, Y.; Okumura, S.; Minakata, S. Angew. Chem., Int. Ed. 2012, 51, 7804 .

[54] (a) Renner, C.; Kusebauch, U.; Loweneck, M.; Milbradt, A. G.; Moroder, L. J. Pep. Res. 2005, 65, 4.

(b) Li, Y. C.; Li, S. H.; Qi, C.; Zhang, H. J.; Zhu, M. Y.; Pang, S. P. Acta Chim. Sinica 2011, 69, 2159 (in Chinese).

(李玉川, 李生华, 祁才, 张慧娟, 朱梦宇, 庞思平, 化学学报, 2011, 69, 2159.)

[55] (a) Gowenlock, B. G.; Richter-Addo, G. B. Chem. Rev. 2004, 104, 3315 .

(b) Defoin, A. Synthesis 2004, 706.

[56] Yu, B. C.; Shirai, Y.; Tour, J. M. Tetrahedron 2006, 62, 10303.

[57] Tie, C.; Gallucci, J. C.; Parquette, J. R. J. Am. Chem. Soc. 2006, $128,1162$.

[58] Berryman, B. O.; Sather, C. A.; Rebek Jr., J. Chem. Commun. 2011, 47(2), 656.

[59] Kubitschke, J.; Näther, C.; Herges, R. Eur. J. Org. Chem. 2010, 5041.

[60] (a) Norikane, Y.; Kitamoto, K.; Tamaoki, N. Org. Lett. 2002, 4(22), 3907.

(b) Wei, W. H.; Tomohiro, T.; Kodaka, M.; Okuno, H. J. Org. Chem. 2000, 65, 8979.

[61] Khan, A.; Hecht, S. Chem.-Eur. J. 2006, 12, 4764.

[62] Wada, S.; Urano, M.; Suzuki, H. J. Org. Chem. 2002, 67, 8254.

[63] Laskar, D. D.; Prajapati, D.; Shandu, J. S. J. Chem. Soc., Perkin Trans. 1 2000, 67

[64] Gowda, S.; Gowda, D. C. Synthesis 2002, 460.

[65] Srinivasa, G. R.; Abiraj, K.; Gowda, D. C. Aust. J. Chem. 2004, 57, 609.

[66] Pasha, M. A.; Jayashankara, V. P. Ultrason. Sonochem. 2005, 12, 433.

[67] Stolarczyk, K.; Bilewick, R.; Skwierawska, A.; Biernat, J. F. J. Inclusion Phenom. Macrocyclic Chem. 2004, 49, 173.

[68] (a)Peng, Y.; Zhao, Z.-G.; Yang, Z.-X.; Mu, Q.-M.; Chen, S.-H. Chin. J. Org. Chem. 2005, 25, 462 (in Chinese).

(彭游, 赵志刚, 杨祖幸, 牟其明, 陈淑华, 有机化学, 2005, 25, 462.)

(b) Altomare, A.; Ciardelli, F.; Marchini, M.; Solaro, R. Polymer 2005, 46, 2086.

(c) Xiong, C.-X.; Niu, Y.-S.; Zhou, W.; Dong, L.-J. J. Chem. Res. 2006, (2), 139.

[69] Srinivasa, G. R.; Abiraj, K.; Gowda, D. C. Tetrahedron Lett. 2003, 44,5835 .
[70] Srinivasa, G. R.; Abiraj, K.; Gowda, D. C. Synth. Commun. 2003, $33,4221$.

[71] Huang, Q. M.; Pan, W.; Li, Q.; Wang, S. W.; Li, Z. Y.; Pan, Z. Q. Chin. J. Org. Chem. 2011, 31, 336 (in Chinese).

(黄齐茂, 潘威, 李清, 王司卫, 李志远, 潘志权, 有机化学, 2011, 31, 336.)

[72] Won, S.; Kim, W.; Kim, H. Bull. Korean Chem. Soc. 2006, 27, 195.

[73] Sakai, N.; Fujii, K.; Nabeshima, S.; Ikeda, R.; Konakahara, T. Chem. Commun. 2010, 46, 3173.

[74] Zhu, H.; Ke, X.; Yang, X.; Sarina, S.; Liu, H. Angew. Chem., Int. Ed. 2010, 49, 9657.

[75] Shimao, I.; Oae, S. Bull. Chem. Soc. Jpn. 1983, 56, 643.

[76] Yamamoto, J.; Nishigaki, Y.; Umezu, M. Tetrahedron 1980, 36, 3177.

[77] Vozza, J. F. J. Org. Chem. 1969, 34, 3219.

[78] Olsen, H.; Snyder, J. P. J. Am. Chem. Soc. 1977, 99, 1524.

[79] Nanjundaswamy H. M.; Pasha, M. A. Synth. Commun. 2005, 35, 2163.

[80] Nanjundaswamy, H. M.; Pasha, M. A. Indian J. Chem. 2006, 45B, 1086.

[81] Konwar, D.; Boruah, R. C.; Sandhu, J. S. Synthesis 1990, 337.

[82] Ilias, M.; Barman, D. C.; Prajapati, D.; Sandhu, J. S. Tetrahedron Lett. 2002, 43, 1877.

[83] Saini, A.; Kumar, S.; Sandhu, J. S. Synlett 2006, 395.

[84] Baik, W.; Kim, D. I.; Koo, S.; Rhee, J. U.; Shin, S. H.; Kim, B. H. Tetrahedron Lett. 1997, 38, 845.

[85] Sanz, R.; Escribano, J.; Fernandez, Y.; Aguado, R.; Pedrosa, M. R.; Arnáiz, F. J. Synlett 2005, 1389.

[86] Olah, G. A.; Balaram Gupta, B. G.; Narang, S. C. J. Org. Chem. 1978, 43, 4503.

[87] Mukaiyama, T.; Nambu, H.; Okamoto, M. J. Org. Chem. 1962, 27, 3651.

[88] Scriven, E. F. V.; Suschitzky, H. Tetrahedron Lett. 1973, 14, 103.

[89] Mankad, P. N.; Muller, P.; Peters, C. J. J. Am. Chem. Soc. 2010, 132, 4083 .

[90] Takaoka, A.; Moret, M.-E.; Peters, C. J. J. Am. Chem. Soc. 2012, 134, 6695 .

[91] Haghbeen, K.; Tan, E. W. J. Org. Chem. 1998, 63, 4503.

[92] Wang, S.-R.; Li, X.-G; He, L.-L.; Hu, Y.-Q. J. Tianjin Univ. 2007, 40, 1295 (in Chinese).

(王世荣, 李祥高, 何莉莉, 胡雅琴, 天津大学学报, 2007, 40, 1295.)

[93] Xu, L. J.; Lin, W. H.; Sun, W. L.; Shen, Z. Q. Acta Polym. Sin. 2008, 1,62 (in Chinese). (徐否杰, 林维红, 孙维林, 沈之荃, 高分子学报, 2008, 1, 62.)

[94] Han, M.; Ishikawa, D.; Muto, E.; Hara, M. J. Lumines 2009, 129, 1163.

[95] Feng, F.; Hu, W. B.; Liu, H. X.; Nie, X. L.; Yu, A. N. Chin. J. Appl. Chem. 2010, 27, 169 (in Chinese). (冯驸, 胡卫兵, 刘红霞, 聂响亮, 余爱农, 应用化学, 2010, 27, 169.)

[96] Liu, G.; Zhang, B.; Chen, Y.; Zhu, C.-X.; Zeng, L. J.; Chan, D. S.-H.; Neoh, K.-G.; Kang, E.-T. J. Mater. Chem. 2011, 21, 6027.

[97] Merrington, J.; James, M.; Bradley, M. Chem. Commun. 2002, 38, 140.

[98] Kim, J. Y.; Kim, G.; Kim, C. R.; Lee, S. H.; Lee, J. H.; Kim, J. S. J. Org. Chem. 2003, 68, 1933.

[99] Barbero, M.; Degani, I.; Dughera, S.; Fochi, R.; Perracino, P. Synthesis 1998, 1235.

[100] Merino, E. Chem. Soc. Rev. 2011, 40, 3835.

(Qin, X.) 